\title{
Dynamic cantilever magnetometry of individual CoFeB nanotubes
}

\author{
B. Gross, ${ }^{1}$ D. P. Weber, ${ }^{1}$ D. Rüffer, ${ }^{2}$ A. Buchter, ${ }^{1}$ F. Heimbach, ${ }^{3}$ A. Fontcuberta i Morral, ${ }^{2}$ D. Grundler, ${ }^{4}$ and M. Poggio ${ }^{1}$ \\ ${ }^{1}$ Departement Physik, Universität Basel, 4056 Basel, Switzerland \\ ${ }^{2}$ Laboratoire des Matériaux Semiconducteur, Institut des Matériaux, Ecole Polytechnique Fédérale de Lausanne, 1015 Lausanne, Switzerland \\ ${ }^{3}$ Lehrstuhl für Physik funktionaler Schichtsysteme, Physik Departement E10, Technische Universität München, 85747 Garching, Germany \\ ${ }^{4}$ Laboratoire des Matériaux Magnétiques Nanostructurés et Magnoniques, Institut des Matériaux, \\ Ecole Polytechnique Fédérale de Lausanne, 1015 Lausanne, Switzerland
}

(Received 30 November 2015; revised manuscript received 19 January 2016; published 5 February 2016)

\begin{abstract}
We investigate single $\mathrm{CoFeB}$ nanotubes with hexagonal cross section using dynamic cantilever magnetometry (DCM). We develop both an analytical model based on the Stoner-Wohlfarth approximation and a broadly applicable numerical framework for analyzing DCM measurements of magnetic nanostructures. Magnetometry data taken at $4.2 \mathrm{~K}$ show the presence of a uniformly magnetized configuration at high external fields with $\mu_{0} M_{s}=1.3 \pm 0.1 \mathrm{~T}$ and nonuniform configurations at low fields. In this low-field regime, comparison between numerical simulations and DCM measurements supports the existence of flux-closure configurations. Crucially, evidence of such configurations is only apparent because of the sensitivity of DCM to single nanotubes, whereas conventional measurements of ensembles are often obscured by sample-to-sample inhomogeneities in size, shape, and orientation.
\end{abstract}

DOI: 10.1103/PhysRevB.93.064409

\section{INTRODUCTION}

Applications ranging from dense magnetic memories [1] to magnetic sensing [2] and imaging [3-5] have motivated the synthesis and study of a wide range of nanometer-scale magnets. At these size scales, geometry plays a crucial role in determining the magnetization configurations that are stable. Ferromagnetic nanotubes are a particularly interesting morphology of nanomagnet, since the lack of a magnetic core can make flux-closure magnetization configurations more favorable than uniform single domain states. These configurations minimize magnetostatic energy and therefore produce minimal stray fields, e.g., reducing interactions between nanomagnets in densely packed magnetic memories. A variety of stable configurations have been predicted at low fields and at remanence, including a global vortex configuration, where the spins point circumferentially around the tube, multidomain states composed of uniform and vortex domains, and an onion state, consisting of two oppositely oriented circumferential domains plus two uniform domains oriented in the direction of the tube axis [6-14]. Flux-closure configurations are particularly interesting since, during magnetization reversal, they avoid the Bloch point structure and thereby result in a fast and reproducible reversal process [9]. There are a variety of theories describing the reversal process for such nanotubes [10,15-19], via a propagating vortex, a transverse domain wall, or a mixed multidomain combination of the former.

Given their small magnetic moment, however, measurements of magnetization and magnetization reversal in ferromagnetic nanotubes have mostly been conducted on large ensembles [16-18,20-23]. Difficultly in controlling the distribution of size, shape, and orientation, as well as the interactions between nanotubes complicate the interpretation of these results. Here, we avoid these complications by investigating individual $\mathrm{CoFeB}$ nanotubes by dynamic cantilever magnetometry (DCM) [24-26]. DCM allows the measurement of individual nanomagnets as a function of applied external field in controlled orientations and provides information on the saturation magnetization, anisotropy, and the switching behavior. The technique has been recently used to measure both normal and superconducting mesoscopic rings [27,28], both individual superconducting and ferromagnetic nanostructures [13,29-31], and the skyrmion phase in a single nanomagnet [32]. We develop a simple analytical model for the DCM of magnetic nanostructures, as well as a numerical framework applicable to a broad range of nanomagnetic samples. Using these tools to guide our interpretation of the data, we find evidence for stable low-field flux-closure configurations and gain insight on the sequence of the magnetization reversal process. We note that the applicability of our numerical DCM model is not limited to ferromagnetic systems and could form the basis for simulating and interpreting DCM measurements in samples with a variety of complex magnetic configurations.

\section{SAMPLES}

The samples in this study are chosen because of their similarity to idealized ferromagnetic nanotubes, which have been the subject of extensive theoretical modeling [9,10,15,33-38]. The fabrication process and choice of material facilitate smooth sample surfaces, a comparatively large saturation magnetization, and avoid magnetocrystalline anisotropy [39,40]. These properties yield strong nanomagnets, whose stable magnetization configurations are determined by their designed geometry, rather than by defects or geometrical imperfections.

The $\mathrm{CoFeB}$ nanotubes consist of a nonmagnetic GaAs core surrounded by a magnetic $\mathrm{CoFeB}$ shell with a hexagonal cross section, as sketched in Fig. 1. To fabricate the samples, GaAs nanowires are grown on a Si (111) substrate using Ga droplets as catalysts by molecular beam epitaxy [39]. Then $\mathrm{CoFeB}$ is magnetron sputtered on the nanowires, producing a homogeneously thick $(t=30 \pm 2 \mathrm{~nm})$, amorphous shell, avoiding magnetocrystalline anisotropy in the samples [41]. The saturation magnetization of planar $\mathrm{CoFeB}$ films of similar thickness as for the present nanotubes has been measured 

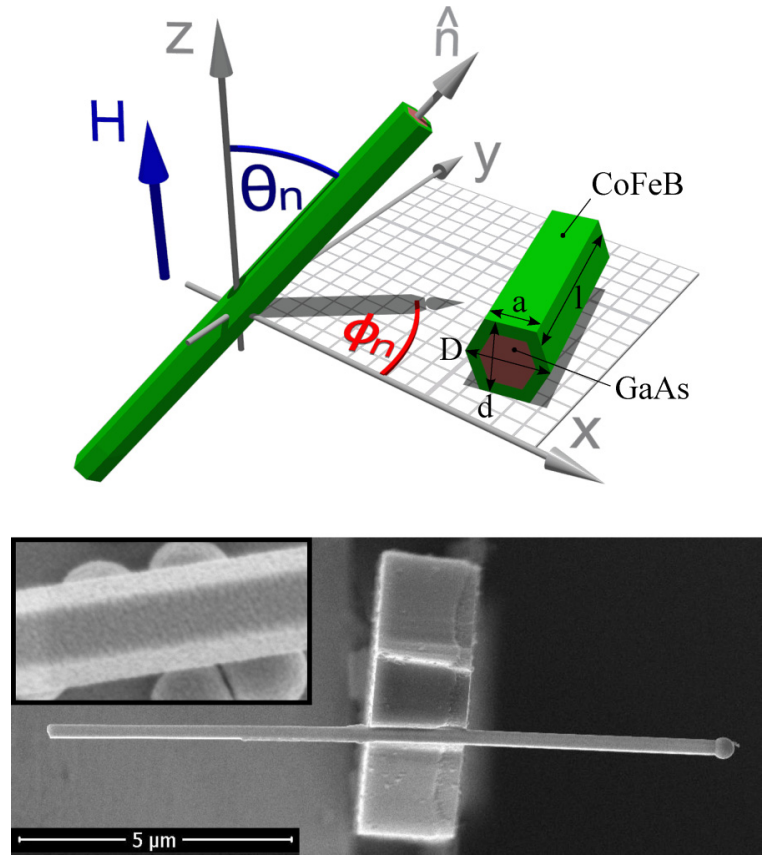

FIG. 1. Top: Illustration of the sample structure and definition of coordinates. The external magnetic field $\mathbf{H}$ defines $\hat{z}$, which coincides with the cantilever axis. Bottom: SEM image of a $\mathrm{CoFeB}$ nanotube attached to the end of a Si cantilever (the long axis of the cantilever is perpendicular to the image plane). Inset: Closeup of a nanotube.

to be $\mu_{0} M_{s}=1.80 \mathrm{~T}$ [42]. The resulting nanotubes are typically over $10 \mu \mathrm{m}$ long and about $250 \mathrm{~nm}$ in diameter. The dimensions of the individual nanotubes used in this study are summarized in Table I as determined using scanning electron microscopy (SEM), cf. Fig. 1 for a representative image. Note that one end of the nanotubes is terminated by the Ga droplet from the nanowire growth process, covered with $\mathrm{CoFeB}$, while at the other end the sample has been broken off the substrate. As a result, the ends of the tubes are not-in general-capped by an open and perfectly flat end. Nevertheless, SEM images reveal continuous and defect-free tubes, whose surface roughness is better than $3 \mathrm{~nm}$ [39]. This near perfection is in contrast to the Ni nanotubes studied by DCM in Weber et al. [31], which contained a peak-to-peak roughness on the order of $10 \mathrm{~nm}$. Buchter et al. [13] showed that the unintentional roughness of these Ni nanotubes likely made them different enough from idealized ferromagnetic nanotubes to result in a magnetization reversal process unlike that predicted by theory.

TABLE I. Dimensions of the measured nanotubes, quantities are defined in Fig. 1. An error of $\pm 0.02 \mu \mathrm{m}$ is estimated for $D, d$, and $a$.

\begin{tabular}{lccccc}
\hline \hline Config. & $l(\mu \mathrm{m})$ & $D(\mu \mathrm{m})$ & $d(\mu \mathrm{m})$ & $a(\mu \mathrm{m})$ & $V\left(10^{-19} \mathrm{~m}^{3}\right)$ \\
\hline 1 & $10.3 \pm 1.0$ & 0.26 & 0.26 & 0.12 & $2.3 \pm 0.6$ \\
2 & $12.6 \pm 0.1$ & 0.27 & 0.24 & 0.15 & $2.8 \pm 0.5$ \\
3 & $12.0 \pm 0.1$ & 0.25 & 0.24 & 0.11 & $2.4 \pm 0.4$ \\
\hline \hline
\end{tabular}

\section{EXPERIMENTAL SETUP}

DCM involves a measurement in an externally applied magnetic field of the mechanical resonance frequency of a cantilever, to which the nanomagnet of interest has been attached. By using ultrasoft, single-crystal Si cantilevers, we achieve a sufficiently high sensitivity to probe the magnetic states of single nanotubes. In order to carry out such measurements, individual $\mathrm{CoFeB}$ nanotubes are glued to the end of a cantilever with epoxy (Gatan G1) using micromanipulators under a customized optical microscope [31]. Three orientations of the nanotubes relative to the applied magnetic field are prepared, as depicted in Fig. 2 and labeled configurations 1, 2, and 3. The error of the actual nanotube orientations relative to the desired orthogonal orientations is $\pm 10^{\circ}$.

The cantilevers used here are about $150 \mu \mathrm{m}$ long, $3.5 \mu \mathrm{m}$ wide, and $0.12 \mu \mathrm{m}$ thick with a mass-loaded end being $18 \mu \mathrm{m}$ long, $4.9 \mu \mathrm{m}$ wide, and $1.7 \mu \mathrm{m}$ thick. As shown in Table II, their fundamental mechanical modes have quality factors $Q$ around $3 \times 10^{4}$, resonance frequencies $f_{0}$ between 2 and $3 \mathrm{kHz}$, and spring constants $k_{0}$ around $50 \mu \mathrm{N} / \mathrm{m}$ under measurement conditions, i.e., in a vacuum chamber with a pressure below $10^{-6}$ mbar in a cryostat with $T=4.2 \mathrm{~K}$.
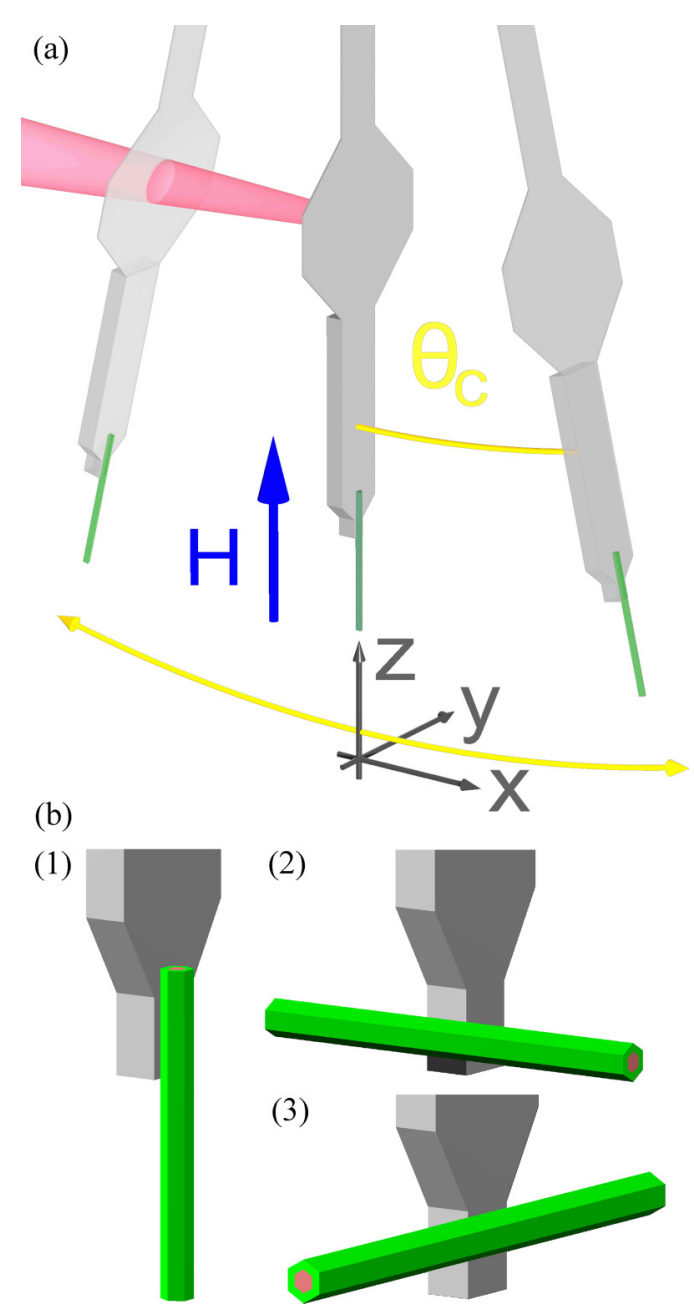

FIG. 2. (a) Schematic diagram of the experimental setup and (b) the three orientations of the nanotubes with respect to the magnetic field, which we label configuration 1, 2, and 3 . 
TABLE II. Properties of the cantilevers used for the experiments.

\begin{tabular}{lcccc}
\hline \hline Config. & $l_{e}(\mu \mathrm{m})$ & $f_{0}(\mathrm{~Hz})$ & $k_{0}(\mu \mathrm{N} / \mathrm{m})$ & $Q$ \\
\hline 1 & $108.2 \pm 0.3$ & 2191.0 & $48.7 \pm 4.5$ & $33 \times 10^{3}$ \\
2 & $107.8 \pm 0.3$ & 2211.9 & $48.5 \pm 4.7$ & $41 \times 10^{3}$ \\
3 & $107.8 \pm 0.3$ & 2107.8 & $40.3 \pm 3.0$ & $36 \times 10^{3}$ \\
\hline \hline
\end{tabular}

A superconducting magnet built into the cryostat allows the application of an external magnetic field up to $\pm 6 \mathrm{~T}$ parallel to the cantilever axis $(\hat{\mathbf{z}})$. The deflection of the cantilever (along $\hat{\mathbf{x}})$ is measured by a fiber interferometer [43] with $100 \mathrm{nW}$ of $1550 \mathrm{~nm}$ laser light focused onto a $11 \mu \mathrm{m}$ wide paddle near the mass-loaded end of the cantilever. A piezo-electric actuator mechanically drives the cantilever at its resonance frequency with a constant oscillation amplitude of $40 \mathrm{~nm}$, using a feed-back loop implemented by a field-programmable gate array. This process of self-oscillation enables the fast and accurate extraction of the resonance frequency from the cantilever deflection signal.

\section{DYNAMIC CANTILEVER MAGNETOMETRY}

The energy of the magnet-on-cantilever system can be described by the sum of a mechanical energy term, related to the cantilever (approximated here as a simple harmonic oscillator), and a magnetic energy term, related to the attached sample:

$$
E=\frac{1}{2} k_{0}\left(l_{e} \theta_{c}\right)^{2}+E_{m},
$$

where $k_{0}$ is the spring constant, $l_{e}$ is the effective length of the cantilever's fundamental mode, $\theta_{c}$ is the angle of the cantilever free end with respect to $\mathbf{H}$, and $E_{m}$ is the magnetic energy. Given that the Si cantilever and the epoxy used to attach the sample have no magnetic response, the magnetic energy depends only on the properties of the attached nanomagnet. As shown in Fig. 2, H sets $\hat{\mathbf{z}}$, while $\hat{\mathbf{y}}$ is coincident with the cantilever's axis of rotation. Therefore the measured cantilever deflection $\theta_{c}$ depends on the component of the torque along $\hat{\mathbf{y}}$, which is given by $\tau_{y}=-\partial E / \partial \theta_{c}$. Since $\theta_{c} \ll 1$ during the measurement (i.e., $x / l_{e} \ll 1$, where $x=l_{e} \theta_{c}$ is the position of the cantilever's free end), we expand $E_{m}$ as a function of $\theta_{c}$ around $\theta_{c}=0$. Keeping only terms up to first order in $\theta_{c}$, we find:

$$
\tau_{y}=-\left(\left.\frac{\partial E_{m}}{\partial \theta_{c}}\right|_{\theta_{c}=0}\right)-\left[k_{0} l_{e}^{2}+\left(\left.\frac{\partial^{2} E_{m}}{\partial \theta_{c}^{2}}\right|_{\theta_{c}=0}\right)\right] \theta_{c},
$$

where $\left.\frac{\partial E_{m}}{\partial \theta_{c}}\right|_{\theta_{c}=0}$ and $\left.\frac{\partial^{2} E_{m}}{\partial \theta_{c}^{2}}\right|_{\theta_{c}=0}$ are the first and second derivatives of the magnetic energy with respect to $\theta_{c}$ at the cantilever's equilibrium angle. The equation of motion for this harmonic oscillator is

$$
m_{e} \ddot{x}+\Gamma \dot{x}=\tau_{y} / l_{e}
$$

where $m_{e}$ is the effective mass of the cantilever, and $\Gamma$ is the cantilever's mechanical dissipation. We then see that the first term in Eq. (2) produces a constant deflection of the cantilever, while the term proportional to $\theta_{c}$ determines the cantilever's spring constant:

$$
\begin{aligned}
& m_{e} \ddot{\theta_{c}}+\Gamma \dot{\theta_{c}}+\left[k_{0}+\frac{1}{l_{e}^{2}}\left(\left.\frac{\partial^{2} E_{m}}{\partial \theta_{c}^{2}}\right|_{\theta_{c}=0}\right)\right] \theta_{c} \\
& =-\frac{1}{l_{e}^{2}}\left(\left.\frac{\partial E_{m}}{\partial \theta_{c}}\right|_{\theta_{c}=0}\right) .
\end{aligned}
$$

Conventional static measurements of cantilever magnetometry keep track of the constant deflection term, while the DCM measurements presented here follow the change in the cantilever spring constant. Using this equation of motion, we solve for the cantilever's frequency shift $\Delta f=f-f_{0}$, where $f$ is the measured resonance frequency and $f_{0}$ is the resonance frequency at $H=0$ (see Mehlin et al. for full derivation [32]):

$$
\Delta f=\frac{f_{0}}{2 k_{0} l_{e}^{2}}\left(\left.\frac{\partial^{2} E_{m}}{\partial \theta_{c}^{2}}\right|_{\theta_{c}=0}\right) .
$$

Note that we have neglected a term in $\Delta f$ which depends on the cantilever dissipation $\Gamma$, which for the cantilevers and samples used here is negligible compared to the magnetic anisotropy term. Measurements of $\Delta f$ thus reveal the curvature of the magnetic energy with respect to sample rotations about the cantilever oscillation axis. By mounting the sample in various configurations, we investigate the energy curvature about the various rotation axes.

In general, a positive value of $\Delta f$ implies that $E_{m}\left(\theta_{c}\right)$ is in a local minimum with respect to $\theta_{c}$, and therefore changing the cantilever angle increases $E_{m}$. In other words, given the alignment of the nanotube's net magnetization, its physical orientation in the $x z$ plane is energetically favorable. Therefore, the cantilever experiences a springlike magnetic restoring force, which stabilizes this orientation. This "magnetic spring" produces an effective hardening of the cantilever spring constant, reflected as an increase in its resonant frequency. On the other hand, a negative value of $\Delta f$ corresponds to a local maximum in $E_{m}\left(\theta_{c}\right)$. That is, given the alignment of the nanotube's net magnetization, its physical orientation in the $x z$ plane is energetically unfavorable. The cantilever thus experiences an antirestoring force, resulting in an effective softening of the cantilever spring constant and thus a reduction in its resonance frequency.

\section{MODELING}

\section{A. Analytical approximation}

In order to establish a framework from which to interpret our DCM measurements, we begin by modeling our ferromagnetic nanotubes as idealized single-domain magnets. In this simplified model, the magnetization is uniform throughout the magnet and rotates in unison. Its direction is determined by its magnetostatic energy, which we reduce to the Zeeman energy and the anisotropy energy. Given the polycrystallinity and large aspect ratio of the nanotubes (roughly 30:1), the latter is dominated by shape anisotropy. In literature [44,45], magnetic anisotropy of small magnetic particles has frequently been addressed by working within the Stoner-Wohlfarth (SW) approximation [46]. For simplicity, particles are modelled as uniformly magnetized, prolate ellipsoids of revolution, 
with a demagnetizing field $\mathbf{H}_{\mathrm{dm}}=-D \circ \mathbf{M}$ produced by the magnetization $\mathbf{M}$, where $\mathbf{H}_{\mathrm{dm}}$ turns out to be homogeneous. $D$ is a tensor consisting of the diagonal elements $D_{x}, D_{y}$, and $D_{z}$, the demagnetization factors, which describe the anisotropy due to the shape of the particle. It can be shown that, in a uniform applied field, the magnetization of a single-domain particle of arbitrary shape behaves precisely as that of a suitably chosen ellipsoid [47]. By applying this generalization, one can derive the magnetometric demagnetization factors and therefore the magnetic behavior for any arbitrary single-domain magnet. Note, however, that for nonellipsoids the homogeneity of $\mathbf{H}_{\mathrm{dm}}$ within the magnet is not preserved.

In order to calculate demagnetization factors for our ferromagnetic nanotubes, we first approximate the nanotubes as hollow cylinders [48], ignoring the hexagonality of their cross section. This may be justified by the large aspect ratio between length and diameter of the tubes and leads to demagnetization factors of $D_{x}=D_{y} \approx 0.498$ and $D_{z} \approx 0.004$ for the tube axis parallel to the $\hat{\mathbf{z}}$ axis. This implies that we consider only uniaxial anisotropy, which can also be described by a unit vector $\hat{\mathbf{n}}$ along the tube axis and an effective demagnetization factor $D_{u}=D_{z}-D_{x}[44,45]$. Effects of the hexagonal cross section, leading to deviations from the uniaxial description, are then discussed in Sec. VI.

Based on this SW model, we then analytically calculate the magnetization and corresponding frequency shift for any orientation of the nanotube $\left(\phi_{n}, \theta_{n}\right)$ in any applied magnetic field, cf. Fig. 1 for the definition of the angles. This approach is similar to that described by others [49-51] and is described in detail in appendix A. Note that the SW model describes the ferromagnetic nanotubes accurately for large applied external fields, in which the nanotubes are forced into uniform magnetization configurations. However, using this model for low applied fields, where nonuniform magnetic configurations exist, is a strong simplification with limited validity. For this regime, we employ micromagnetic simulations, discussed in the subsequent section, to describe the behavior of the magnetization configurations and the resulting DCM signal. Nevertheless, comparing measurements to the SW model serves as an indicator of the extent to which magnetic configurations are uniform and magnetization reversal is coherent.

\section{B. Numerical calculations}

The limitations of any model for DCM based on the SW approximation are clear: Multidomain magnets or magnets having nonuniform magnetization configurations cannot be described. For this reason, we also carry out hybrid finite/boundary element simulations using the software package Nmag [52], allowing us to model the magnetization distribution within a nanotube. From these simulations we then calculate the DCM frequency shift.

Nmag determines the magnetization distribution step-bystep for each external field value by numerically solving the Landau-Lifschitz-Gilbert equation. We model our nanotubes as perfectly hexagonal tubes with an inner diameter of $190 \mathrm{~nm}$ and a thickness of $30 \mathrm{~nm}$, with an alignment to the external magnetic field that can be freely chosen. Our computational capacity limits us to tubes of $1.5 \mu \mathrm{m}$ length, when keeping the mesh cell size below about $8 \mathrm{~nm}$.

By employing periodic boundary conditions (PBCs), this length limitation can be overcome. This choice, however, implies that the effects of the tube ends are not included in the simulations. PBCs preclude the modeling of end states such as vortex configurations, which nucleate at the tube ends and which have been predicted to initiate magnetization reversal $[9,33]$.

We therefore carry out two types of simulations: the first with $1.5 \mu \mathrm{m}$ long tubes without PBCs and the second with 200 repetitions of a $150 \mathrm{~nm}$-long segment with PBCs. DCM frequency shift is calculated from the Nmag simulations using a procedure discussed in appendix $\mathrm{B}$. We set the exchange coupling constant to $A=28 \mathrm{pJ} / \mathrm{m}$ [53] and use the saturation magnetization $M_{s}$ as the only fit parameter. The initial value for $M_{s}$ is extracted from fits to our high-field DCM measurements using our analytical SW model. Since the modeled nanotubes are shorter than those measured, for comparison with the measurements, we scale the calculated $\Delta f$ proportionally with the ratio of volume of the measured and simulated tubes. The measured volume, and therefore this ratio, is determined by measuring the geometry of the nanotubes, as discussed in Sec. VI. After a few iterations altering $M_{s}$ to optimize agreement with the experimental DCM data at high field, this procedure allows us to extract a value for $M_{s}$.

\section{EXPERIMENTAL RESULTS}

Each $\mathrm{CoFeB}$ nanotube is a complex magnetic system consisting of roughly $10^{10}$ spins. A variety of spin configurations other than the simple macrospin configurations described by the SW approximation can be expected to occur during a field sweep [6,7,9,12-14,37]. Nevertheless, for high fields, at which the Zeeman term dominates over interaction terms in the magnetic energy, treating the system as a single macrospin is valid. In the following, we first analyze this high-field regime and then turn to low fields, where the SW model begins to break down. For that regime, we rely on micromagnetic simulations for further insight.

\section{A. The high-field limit}

In Fig. 3, we plot the measured DCM frequency shift in a field range of $\pm 6 \mathrm{~T}$ for the three different configurations of the nanotubes as depicted in Fig. 2. Data points taken while $H$ is swept in the positive direction are red, while points taken for negative sweeps are blue. To fit the data with the model developed in appendix A for the frequency shift of a SW magnet, we use $k_{0}$ as determined from thermal noise spectra, $l_{e}$ from finite element simulations [54], and $V$ approximated from SEM images. The demagnetization factor $D_{u}=D_{z}-D_{x}$ is calculated by using the geometry of each nanotube measured from SEM images, approximating it as a hollow cylinder, and following the method of Beleggia et al. [48]. $f_{0}$ can be extracted from the measurements of the cantilever resonance frequency at $H=0$. The saturation magnetization $M_{s}$ is used as a fit parameter. The orientation of the nanotubes in the three different configurations, intended to be $\left(\theta_{n}, \phi_{n}\right)=\left(0^{\circ}, 0^{\circ}\right)$, $\left(90^{\circ}, 0^{\circ}\right)$, and $\left(90^{\circ}, 90^{\circ}\right)$, cannot be expected to be perfect 


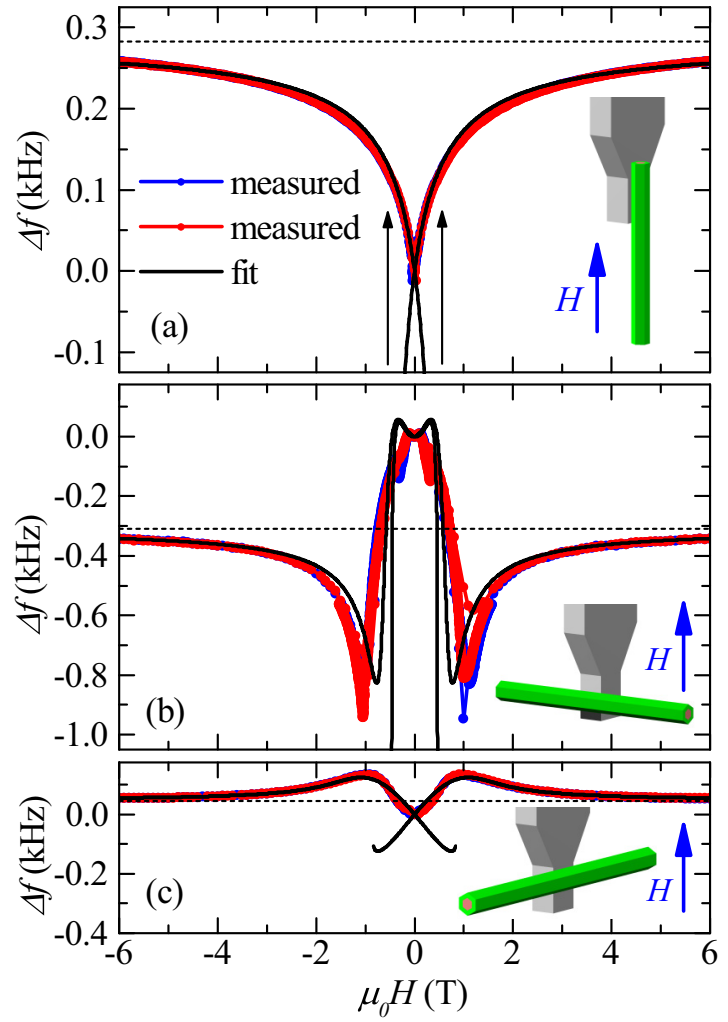

FIG. 3. (a), (b), and (c) frequency shift $\Delta f$ vs $\mu_{0} H$ for configuration 1, 2, and 3 of the nanotubes, respectively. Red (blue) curves are for field sweeps in positive (negative) direction and black curves are SW-fits using the parameters given in Table III. Dashed lines are horizontal asymptotes.

due to the manual attachment of the tubes to the cantilever. Therefore, $\theta_{n}$ and $\phi_{n}$ are also used as fit parameters within a $\pm 10^{\circ}$ range of the intended angles, as estimated from optical and SEM images. The resulting curves (black) are plotted with the experimental data in Fig. 3. The relevant cantilever properties in Table II and the corresponding fit parameters are summarized in Table III. Horizontal asymptotes are depicted as dashed black lines.

Configuration 1, cf. Fig. 3(a), shows a horizontal asymptotic behavior for large fields, where the asymptote is approached from lower values. Data from positive and negative sweep directions coincide very well, and the SW fit gives a good match with the measurement. In this orientation, the nanotube behaves like an SW magnet everywhere except near the lowfield magnetization reversal, as will be discussed in Sec. VI B. From the fit to the analytical model, we extract a saturation

TABLE III. Parameters for fitting $\Delta f$ for the three configurations of the $\mathrm{CoFeB}$ nanotubes. $D_{x}, D_{y}$, and $D_{z}$ are used as fixed parameters, while $M_{s}, \theta_{n}$, and $\phi_{n}$ are free parameters.

\begin{tabular}{lcccrc}
\hline \hline Config. & $D_{x}=D_{y}$ & $D_{z}$ & $\theta_{n}\left({ }^{\circ}\right)$ & $\phi_{n}\left({ }^{\circ}\right)$ & $\mu_{0} M_{s}(\mathrm{~T})$ \\
\hline 1 & 0.4977 & 0.0045 & 3 & 90 & $1.29 \pm 0.18$ \\
2 & 0.4981 & 0.0037 & 86 & 5 & $1.21 \pm 0.12$ \\
3 & 0.4981 & 0.0038 & 80 & 90 & 2.5 \\
\hline \hline
\end{tabular}

magnetization $\mu_{0} M_{s}=1.29 \pm 0.18 \mathrm{~T}$ and orientation angles $\theta_{n}=3^{\circ}$ and $\phi_{n}=90^{\circ}$, which lie within the reasonable range.

Finite element simulations are also carried out according to appendix B for an optimal high-field fit to the measurement. Using the same orientation angles as for the SW model, the simulations yield $\mu_{0} M_{s}=1.32 \pm 0.18 \mathrm{~T}$. Note that the numerical simulations are completely independent of the calculated demagnetization factors and depend only on the exchange coupling constant $A$ and the geometry of the nanotube. The high-field agreement between the SW model and the simulations provides confirmation of the extracted value for $M_{s}$ and the single-domain behavior of the nanotubes at high fields. Further confirmation is provided by a separate DCM measurement in configuration 1 of a different nanotube from the same growth batch. These data show the same qualitative behavior and a fit to the analytical model results in a saturation magnetization $\mu_{0} M_{s}=1.37 \pm 0.24$, which agrees with the first measurement within the error.

For configuration 2, we find a similarly good agreement between data and analytical fit function in the high-field regime. The saturation magnetization $\mu_{0} M_{s}=1.21 \pm 0.12 \mathrm{~T}$ extracted from this measurement is in agreement with that extracted from configuration 1. Furthermore, $\theta_{n}=86^{\circ}$ and $\phi_{n}=5^{\circ}$ again lie within the reasonable range. The corresponding micromagnetic simulations yield $\mu_{0} M_{s}=1.24 \pm 0.12 \mathrm{~T}$ for an optimal high-field fit, which is once again equal to the value extracted from the SW model within our error. In this orientation, the behavior of the nanotube magnetization begins to deviate from the SW model as the SW magnetization begins to coherently rotate toward the $x y$ plane. While the qualitative features of $\Delta f$ displayed by the nanotube and the SW model are the same, as will be discussed in Sec. VI B, the differences indicate a magnetization not executing an idealized coherent rotation.

The third configuration gives a less conclusive picture. Although, as shown in Fig. 3(c), a fit can reproduce the behavior of the data for high fields with reasonable values of $\theta_{n}$ and $\phi_{n}$, it results in an anomalously high saturation magnetization $\mu_{0} M_{s}=2.5 \mathrm{~T}$. This value is larger both than the values extracted in the other orientations and the value known for a planar thin film $\mathrm{CoFeB}$ of $1.8 \mathrm{~T}$ [42]. The largest source of error in our determination of $M_{s}$ comes from our measurement of the nanotube volume $V . V$ is determined by measuring the outer geometry of the nanotubes using SEM images and measuring the mean CoFeB shell thickness from TEMs of representative nanotubes. The uncertainty in $V$ results in an error in $M_{s}$ of nearly $\pm 10 \%$. Nevertheless, such a measurement uncertainty is not large enough to explain the anomalous $M_{s}$ extracted from configuration 3.

A likely explanation for the failure of this fit is that our simplified cylindrical model does not take into account magnetic anisotropies in the plane of the nanotube's hexagonal cross section. In fact, for a perfectly aligned tube in this orientation, the uniaxial anisotropy axis $\hat{\mathbf{n}}$ coincides with the cantilever oscillation axis $\hat{\mathbf{y}}$. As a result, according to our uniaxial model, there should be no variation of $E_{m}$ with respect to $\theta_{c}$ and therefore no frequency shift, as shown by the blue curve in the bottom right graph of Fig. 9. Any detected $\Delta f$ stems from either a misalignment of the nanotube or from deviations of the real sample from a cylindrically symmetrical 
SW tube. In particular, the hexagonal symmetry of the real sample plays an important role in this configuration, given that it produces a curvature of $E_{m}$ about $\hat{\mathbf{n}}$. The magnetic energy of the nanotube cannot, in fact, be fully described by a uniaxial anisotropy, but requires further axes. In configurations 1 and 2 the uniaxial model is appropriate because the anisotropy related to $\hat{\mathbf{n}}$ overwhelms all others in the plane measured by DCM. In configuration 3, however, anisotropies in the cross section of the nanotube dominate and the anisotropy related to $\hat{\mathbf{n}}$ only contributes to $\Delta f$ in the case of misalignment of the sample. Since our model does not take into account the anisotropy in the nanotube cross section, it underestimates the effective anisotropy. As can be seen from the behavior of the high-field asymptote in Eq. (A7), an underestimate of $D_{u}$ then results in the extraction of an anomalously large $M_{s}$ from the fits, just as observed. Given the difficulty of disentangling the effects due to misalignment and additional cross-sectional anisotropies, we abandon a detailed analysis of configuration 3 using the SW model.

Finite element simulations of $\Delta f(H)$ are carried out in this configuration and optimized to match measurements at high field. These numerical calculations yield $\mu_{0} M_{s}=1.25 \mathrm{~T}$ for a large but possible misalignment of $\theta_{n}=70^{\circ}$ and $\phi_{n}=$ $90^{\circ}$. Nevertheless, modeling this configuration remains problematic given the measurement's sensitivity to anisotropies determined by misalignment and the precise cross section of the nanotube. Imperfections in the form of asymmetries in the hexagonal cross section along the nanotube's length could cause significant deviations between the behavior of the real sample and the idealized model. In configurations 1 and 2, the effect of such imperfections causes smaller discrepancies between measurement and model given that the measured magnetic confinement $\left(\partial^{2} E_{m} / \partial \theta_{c}^{2}\right)$ is dominated by the large aspect ratio of the nanotubes rather than their precise shape. For these reasons, we focus the rest of our analysis on the experiments carried out in configurations 1 and 2 .

Finally, the reason for the discrepancy between the saturation magnetization determined from configurations 1 and 2, $\mu_{0} M_{s}=1.3 \pm 0.1 \mathrm{~T}$, and the value reported for twodimensional $\mathrm{CoFeB}$ films of similar thickness, $1.80 \mathrm{~T}$ [42], remains unclear. Errors in the determination of $V$ and $k_{0}$ are not large enough to explain this mismatch. Material degradation through the formation of an outer oxidation layer is also insufficient to explain the reduction in saturation magnetization. About $15 \mathrm{~nm}$ of the $30 \mathrm{~nm}$ magnetic shell would have to oxidize in order to account for the difference, while $\mathrm{x}$ ray absorption spectroscopy (XAS) measurements indicate the presence of an oxide no thicker than $5 \mathrm{~nm}$. Annular dark field (ADF) scanning transmission electron microscopy (STEM) of nanotubes produced under identical conditions shows local variations in the density of the material [39], possibly caused by directional deposition. We are left to conclude that the reduced saturation magnetization with respect to planar films is the result of such variations or some combination of all the aforementioned effects.

\section{B. The low-field limit}

We now analyze the data for low applied magnetic fields, where "low" specifies the field regime in which the
SW model does not reproduce the experimental behavior. From this deviation, it is clear that more complex magnetic configurations than a collective of parallel, coherently rotating spins occur in this regime.

In literature, several nontrivial magnetic configurations are suggested to play a role in magnetization reversal for core-free systems such as the measured nanotubes. For the field parallel to the tube axis there is the "twisted bamboo" state, where two vortex states form at the ends of the tube with a domain parallel to the axis between them $[7,9,13,36,37]$. The magnetization reversal is thought to take place by a propagating vortex or a transverse domain wall [9,10,15,17-19]. For a field applied perpendicular to the nanotube axis, Rüffer et al. [12] suggest the presence of an "onion state." This configuration consists of two oppositely oriented circumferential domains separated by two domain walls, with the latter aligned parallel to the easy anisotropy axis and antiparallel to each other.

The existence of such magnetic configurations has been predicted by both analytical and numerical calculations [6-14]. So far, however, magnetic imaging of the configurations with sufficient spatial resolution to clearly identify the states has not been possible. Techniques that may provide sufficient resolution in the future include scanning SQUID magnetometry, scanning diamond nitrogen-vacancy (NV) center magnetometry [55], magnetic force microscopy (MFM) [6,56], and x-ray circular dichroism-photoelectron emission microscopy (XMCD-PEEM) [11,57].

Our DCM measurements cannot unambiguously determine the magnetic configurations involved in the magnetization reversal. Nevertheless, guided by the SW model and micromagnetic simulations of the DCM response, DCM yields important information about the progression of magnetic configurations as a function of external field. In particular, given a particular progression of magnetic configurations suggested by analytical or numerical calculations, DCM can be used to support or rule out the hypothesis.

\section{Field applied parallel to nanotube axis}

a. DCM measurements. For configuration 1, we plot in Fig. 4(a) the same data as in Fig. 3, focusing on the low magnetic field range. Here, as for high fields, the fit based on the SW model agrees well with much of the measurement. The alignment of the easy axis with the applied field direction combined with the strong shape anisotropy of the nanotube keeps all the magnetic moments parallel to each other and aligned with $\hat{\mathbf{z}}$ for the majority of the magnetic field range. Despite this agreement, magnetization reversal takes place at $\pm 30 \mathrm{mT}$, rather than the $\pm 550 \mathrm{mT}$ predicted by the $\mathrm{SW}$ model. Also, a slight asymmetry in the reversal fields and the switching behavior is likely due to exchange coupling produced by a thin antiferromagnetic native oxide on the nanotube surface [58]. Most notably, however, the reversal takes place over three distinct stages as shown in Fig. 4(a): an initial steplike feature, followed by a plateau near $\Delta f=0$, ending with a final irreversible magnetization switch. Measurements of minor DCM hysteresis loops show that both the initial and final steps are irreversible. In order to visualize the hysteresis curve more clearly, we use Eq. (A9) to extract the effective macrospin magnetization of the nanotube along $\hat{\mathbf{z}}$ from the low-field 


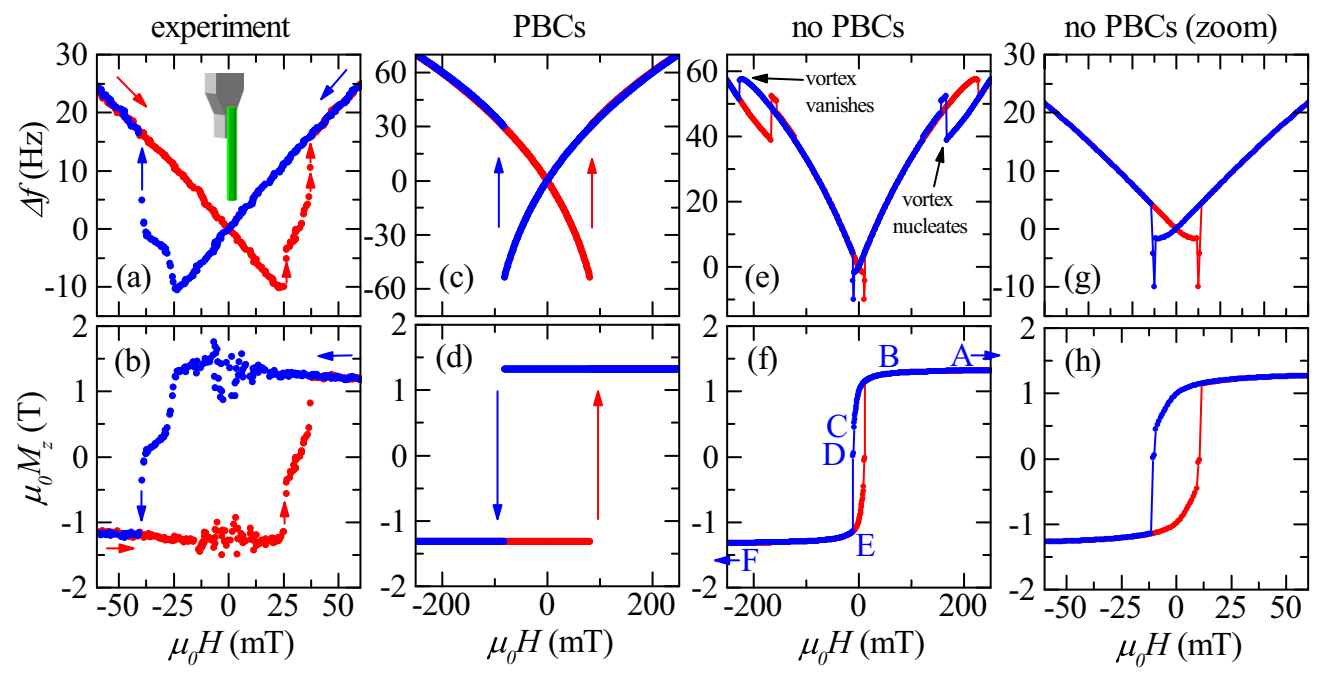

FIG. 4. Frequency shift $\Delta f$ and magnetization $\mu_{0} M_{z}$, in the top and bottom rows respectively, vs $\mu_{0} H$ for nanotube configuration 1 , cf. Fig. 2. Red (blue) curves are measurements for field sweeps in positive (negative) field direction. The first column shows DCM measurements, the second numerical simulations with PBCs, the third numerical simulations without PBCs, and the fourth a zoom of column three.

frequency shift data. The resulting low-field hysteresis curve in Fig. 4(b) shows a square loop with a stable region showing an effective magnetization of nearly zero in the middle of both reversals.

This DCM hysteresis curve differs from that observed for a single $\mathrm{Ni}$ nanotube by Weber et al. [31], where magnetization reversal proceeded through a series of statistically occurring steps attributed to multidomain states. Subsequent measurements by Buchter et al. [13] indicated that the $\mathrm{Ni}$ nanotube samples were separated in roughly $0.5 \mu \mathrm{m}$ long magnetic segments due to the roughness of the film. In our case, the smoothness of the $\mathrm{CoFeB}$ film and the absence of statistically occurring steps in the magnetic hysteresis indicate that the $\mathrm{CoFeB}$ nanotubes are close to ideal ferromagnetic tubes. Note that a hysteresis curve of the same form, with a similar plateau near $\Delta f=0$, is reproduced by a DCM measurement in configuration 1 of a different $\mathrm{CoFeB}$ nanotube from the same growth batch.

b. Numerical simulations. To establish a framework by which this hysteresis curve can be understood, we analyze our micromagnetic simulations. In Figs. 4(c), 4(e), and 4(g), we plot the frequency shift obtained from the simulation of a nanotube calculated with and without PBCs. In both cases, as discussed in Sec. V B, we model a nanotube with a cross section matching the measured tubes and a shorter length dictated by computational limitations. The simulated $\Delta f$ is then scaled up by the ratio of the measured and simulated length of the nanotube $(\Delta f \propto V)$ and $M_{s}$ is chosen for an optimal high-field fit to the measurements, as described in Sec. VI A. Figures 4(d), 4(f), and 4(h) show the magnetization along $\hat{\mathbf{z}}$ corresponding to these simulations.

For simulations calculated with PBCs, $M_{z}$ performs a perfect rectangular hysteresis, and magnetization reversal takes place at $\pm 100 \mathrm{mT}$, which is closer to the experimental value than obtained from the SW model. The magnetization distribution never shows any significant $M_{x}$ and $M_{y}$ components throughout the hysteresis curve, and there is no trace of a plateau regime around $M_{z}=0$. In fact, inspection of the simulated magnetization configurations reveal only the two axially saturated states throughout the hysteresis loop without the appearance of any nonuniform configurations. Just as the SW approximation, this model, which ignores the effects of the nanotube ends, is inadequate for describing the observed low-field behavior.

The simulations calculated without PBCs show a magnetization reversal characterized by the formation of two oppositely oriented vortices nucleating at the two ends, just as in similar simulations carried out by Buchter et al. [13] for $\mathrm{Ni}$ nanotubes. Figures $5(\mathrm{~A})-5(\mathrm{~F})$ show the calculated

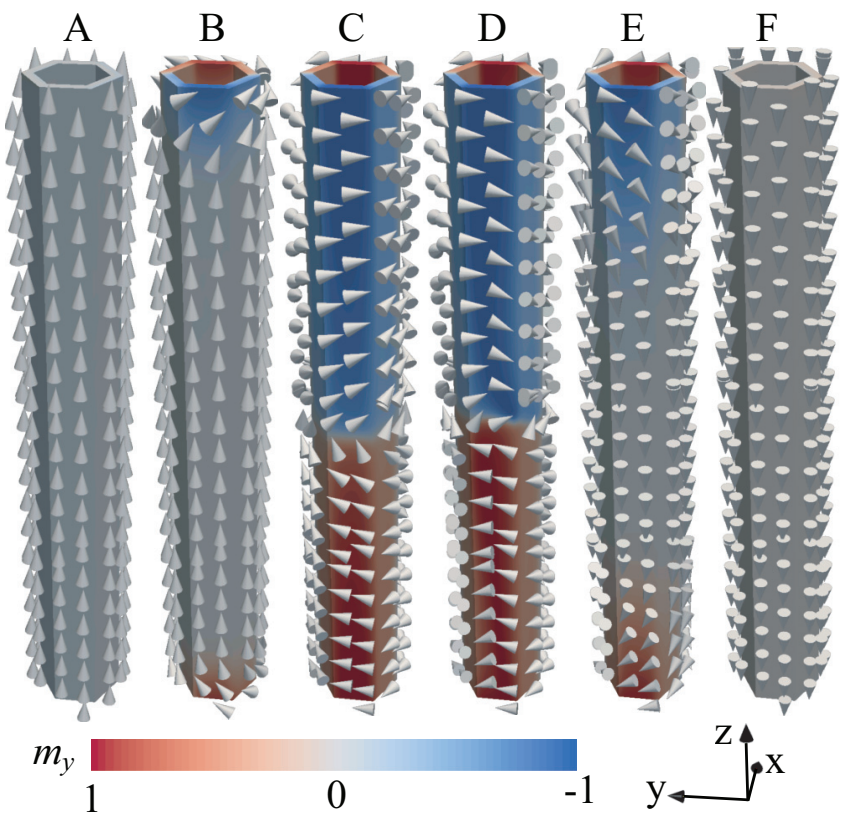

FIG. 5. Visualization of the magnetization configurations calculated without PBCs for 6 different applied magnetic fields indicated in Fig. 4(f). The applied field is swept from pointing in $+\hat{\mathbf{z}}$ to $-\hat{\mathbf{z}}$ with $\hat{\mathbf{n}}$ pointing nearly along $\hat{\mathbf{z}}$ (configuration 1 ). 
magnetization distribution, for various points in the hysteresis loop indicated in Fig. 4(f). Coming from large, positive applied field, following the dark blue data points, all spins are aligned uniformly along the easy axis of the nanotube (A). At around $170 \mathrm{mT}$, oppositely oriented vortices nucleate at the two ends due to the demagnetization effect of the end surfaces (B). This is accompanied by an extremely small jump in the hysteresis curve in Fig. 4(f), which, however, has a visible effect on $\Delta f$, cf. Fig. 4(e). The vortices expand towards the center of the tube when approaching zero field, separated by a Néel domain wall with a positive $M_{z}$ component (C). This configuration is the so-called "twisted bamboo" state alluded to at the beginning of this section. After crossing zero field, this magnetic configuration persists, although $\Delta f<0$ due to the sign change in $H$. At this point, $\Delta f$ approaches zero as the system reaches a configuration with nearly zero net magnetization along $\hat{\mathbf{z}}$ (D). The magnetization in the central part of the tube then undergoes an irreversible switch, corresponding to a discontinuous retreat of the vortices to the ends and an expansion of the central axial region $(\mathrm{E})$. Finally, the vortices disappear at around $-220 \mathrm{mT}(\mathrm{F})$. A clear signature of this vortex mediated magnetization reversal is the discontinuity in $\Delta f$ when the vortices (dis)appear and the rounding of $M_{z}$ close to the coercive field, which is also evident in $\Delta f$, cf. Fig. 4(e). The simulations show no steplike structure in the hysteresis near zero field.

c. Discussion. The simulations calculated without PBCs capture the overall features of the measured DCM and show the formation of a flux-closure configuration near reversal. They do not, however, reproduce the steplike feature in the measured reversal. Also note that the signatures of vortex nucleation in $\Delta f$, which are clearly present in the simulations, are not observed in the measurement. This discrepancy is likely due to the disproportionately large weight of the nanotube ends in the simulation. The simulations are based on a $1.5 \mu \mathrm{m}$ tube, whose DCM response has been scaled up to match the response of the greater than $10 \mu \mathrm{m}$ long measured tube. As a result, the fraction of the magnetic volume occupied by the ends is disproportionately large in the simulated tube in comparison with the measured tube. It is therefore possible that the effect of the vortex nucleation on the measured $\Delta f$ is too weak to clearly appear.

In the future, such discrepancies may be cleared up by increasing computational power and simulating tubes as long as those that are measured. Conversely, improved sample preparation techniques may allow the measurement of tubes shorter than $1.5 \mu \mathrm{m}$. For now, we are left to conclude that simulations showing reversal via flux-closure configurations most closely describe the measured reversal in a parallel applied field. Although we measure a steplike feature in the magnetization hysteresis reminiscent of a stable low-field flux-closure configuration, this feature is not reproduced by the simulations.

Nevertheless, we note that the steplike feature is similar to that observed in magnetization curves of Co rings hosting a stable low-field flux-closure configuration [56,59]. This resemblance suggests the possibility of a stable low-field fluxclosure configuration in the measured nanotubes. At low fields, the single-domain configuration pointing along the nanotube's long axis is dominated by its magnetostatic self-energy. On the other hand, a flux-closure configuration, such as a single vortex or the "twisted bamboo" configuration shown in Figs. 5(C) and 5(D), has reduced magnetostatic self-energy and an increased exchange energy. For a magnetic nanotube, however, the contribution of the vortex configuration to the exchange energy is small: The absence of the magnetic core precludes the formation of a central vortex, which, in nanowires for example, provides a large contribution to the exchange energy. As a result of this reduced exchange contribution in nanotubes, a flux-closure configuration can be the lowest energy state and thus the stable configuration at low fields.

We speculate that imperfections in the real sample-not included in the simulations-could alter the hysteresis and stabilize a low-field magnetic configuration. Possible imperfections include the nonideal termination of the nanotube ends, an antiferromagnetic native oxide layer, magnetic pinning sites, or a growth-induced magnetic anisotropy [60]. However, while a flux-closure configuration is among the possibilities explaining the steplike feature in the hysteresis, other configurations with no net magnetization along $\hat{\mathbf{z}}$ cannot be excluded, e.g., segments of opposing uniaxially aligned domains.

\section{Field applied perpendicular to nanotube axis}

a. DCM measurements. For configurations 2 and 3, the magnetization should execute the same progression as a function of $H$, since the external field is in both cases applied perpendicular to the nanotube long axis. In each orientation, however, we probe the anisotropy in a different plane of the nanotube leading to a different $\Delta f(H)$. We focus exclusively on an analysis of configuration 2 , given the difficulty disentangling the effects of sample misalignment from the anisotropy in the nanotube cross section as discussed in Sec. VI A.

For $\mu_{0}|H| \gtrsim 1 \mathrm{~T}$, the fit based on the SW model describes the data well, as discussed in Sec. VI A. This agreement shows the presence of a single-domain configuration with all magnetic moments pointing along the field direction, cf. the last column of charts in Fig. 9. In the SW model, the two minima of $\Delta f$ mark the field magnitude, below which $\mathbf{M}$ starts rotating towards the long axis of the nanotube. This minimum occurs at $\mu_{0}|H| \approx 1 \mathrm{~T}$ for the measurement. In Figs. 6(a) and 6(b) we show the data for this lower field regime, where the coherent $\mathrm{SW}$ reversal is superseded by a more complex behavior. In this regime a double hump feature is observed in $\Delta f(H)$, which shows hysteretic behavior and can include either a discontinuous jump close to one of the humps [see Fig. 6(b)] or three discontinuous spikes between $0.5 \mathrm{~T}$ and 1.0 T [see Fig. 6(a)]. These two different types of $\Delta f(H)$ curves occur statistically and, though qualitatively similar, include consistent differences for $\mu_{0}|H| \lesssim 1 \mathrm{~T}$.

b. Numerical simulations. In Figs. 6(c) and 6(d) we compare the measured curves for decreasing magnetic field (blue) with the results from the finite element simulations (black) and find an overall agreement between the curves where all major features are reproduced. The low-field double hump feature is well reproduced in both the simulations with and without PBCs, especially in comparison with the poorly matching low-field $\Delta f(H)$ predicted by the SW model, shown in Fig. 3(b). We therefore identify this low-field feature as an 


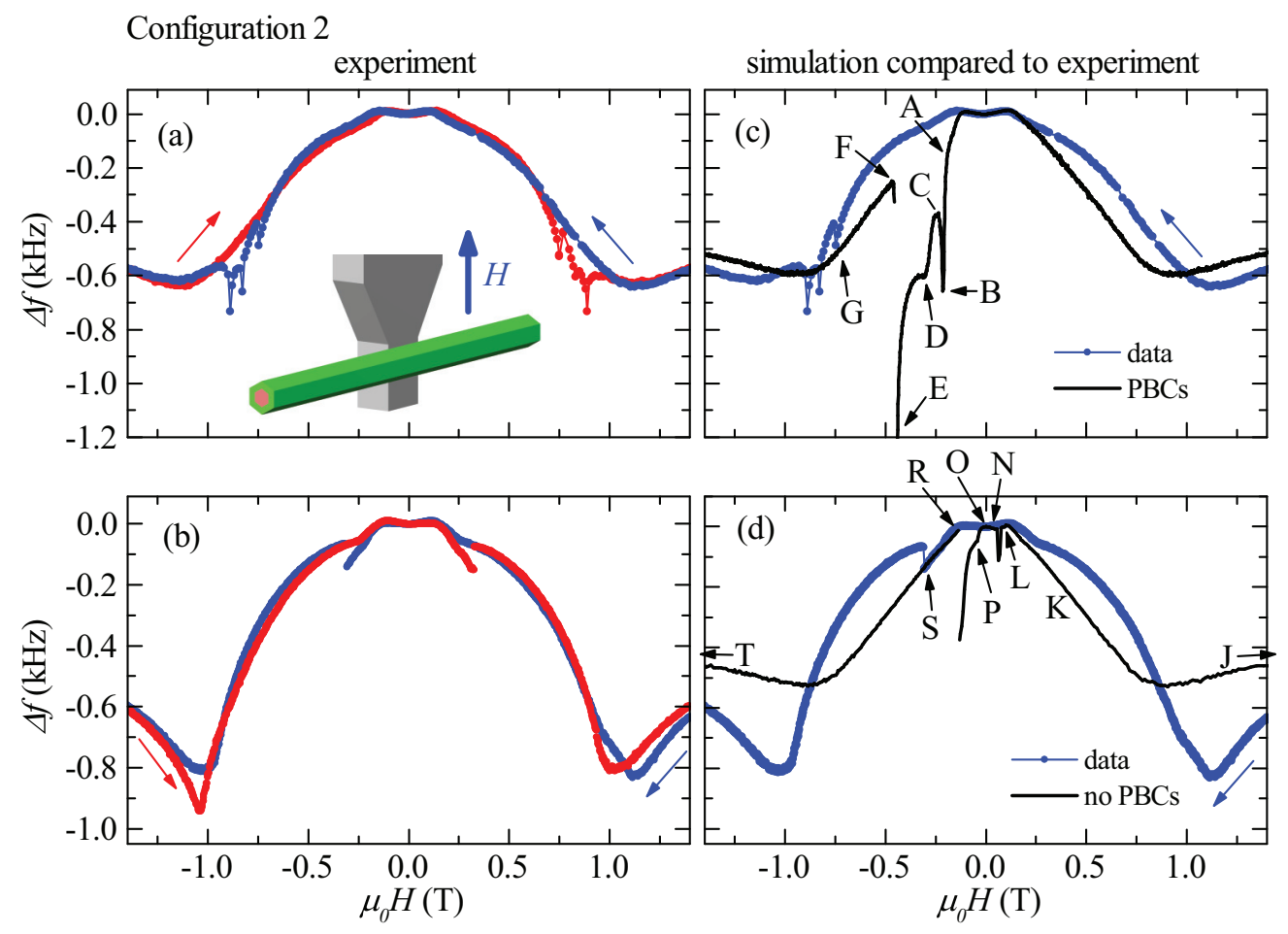

FIG. 6. Frequency shift $\Delta f$ vs $\mu_{0} H$ for nanotube configuration 2, cf. Fig. 2. Red (blue) curves are measurements for field sweeps in positive (negative) field direction. Two different types of hysteresis loops are observed to occur statistically, depicted in (a) and (b). (c) compares the measured data from (a), with simulations calculated with PBCs in black. (d) compares the measured data from (b) with simulations calculated without PBCs in black.

effect of the hexagonality of the nanotube's cross section, which is absent from the SW model and present in both numerical simulations.

Interestingly, the discontinuities observed for the progression shown in Fig. 6(b) and for the simulated nanotube without PBCs are similar, while the ones observed for the progression shown in Fig. 6(a) resemble those seen in the simulations with PBCs. The latter shows three strong spikes after passing zero field, when coming from positive field, that are also observable in experiment, although weaker. As discussed in the following, we find that all these discontinuities arise due to an interplay between the imperfect alignment of the nanotubes and their hexagonal cross section.

As shown in Fig. 2(d), in both configurations 2 and 3, the hexagon is aligned such that its two lateral facets are parallel - or in the usual case of slight misalignment, nearly parallel - to the applied field. Within such a hexagonal cross section, the demagnetization field opposing the external field varies strongly as a function of position, cf. Fig. 7(I). As a result, sweeping an external field applied perpendicular to the long axis of the nanotube down from large values, moments in the top and bottom vertices of the hexagon will start rotating toward the long axis before moments in other locations. Moments in the four top and bottom facets will next begin rotating, while moments in the two side facets will rotate at the smallest fields. As the field is increased from zero again, this staggered rotation occurs in reverse order.

Furthermore, given the inevitable misalignment of $\theta_{n}$, the magnetic moments prefer to rotate towards one of the two easy axis directions, when coming from large fields: e.g., towards $\hat{\mathbf{n}}$ rather than $-\hat{\mathbf{n}}$ coming from positive $H$ in Fig. 1. This preferred direction depends on the direction from which the field is swept, i.e., misalignment from perfect perpendicularity with $\mathbf{H}$ introduces hysteresis. Therefore, at

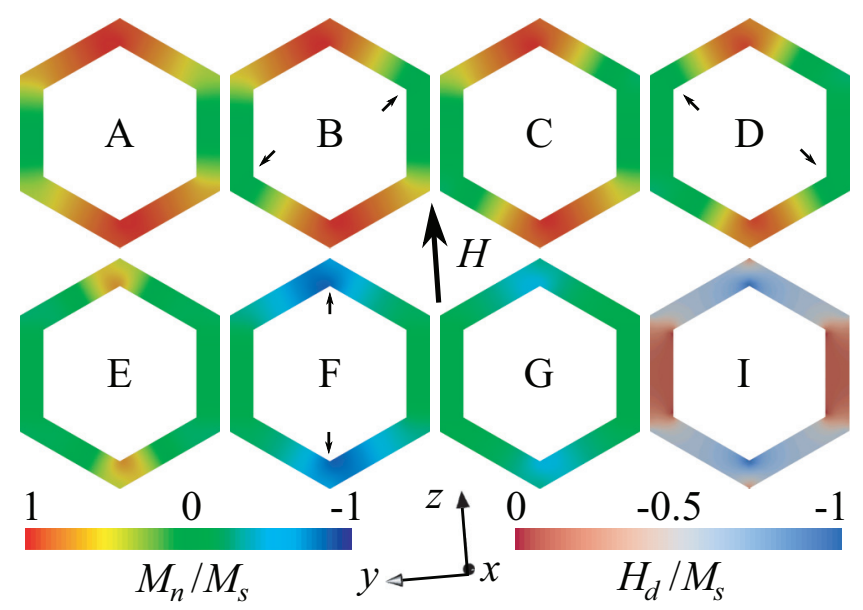

FIG. 7. Simulated magnetization $M_{\hat{n}} / M_{s}$ with PBCs. Images (A)-(G) depict the $M_{\hat{n}}$ component of magnetization in the nanotube cross section for the corresponding field values indicated in Fig. 6(d). Image I shows the local demagnetizing field in $\hat{\mathbf{z}}$ direction $H_{d} / M_{s}$ for an applied field of $H=1.15 M_{s}$. In all cases the applied field $\mathbf{H}$ is slightly misaligned from being perpendicular to the nanotube long axis, as shown by the arrow. $\theta_{n}=86^{\circ}$ and $\phi_{n}=5^{\circ}$ as extracted from the high-field SW fits for data obtained in configuration 2, c.f. Table III. 
zero field, the magnetization tends to point along the easy axis direction preferred by the sample's magnetic history. Upon reversal of the applied field direction, the other easy axis direction becomes energetically favorable, ultimately resulting in a discontinuous reversal. This reversal along the easy axis produces the discontinuities observed in both DCM measurements and simulations.

With this insight, we now follow the simulated $\Delta f(H)$ curve calculated with PBCs in Fig. 6(c), which most closely matches the behavior measured in Fig. 6(a). As the field is swept down from positive fields near zero, the magnetization tends to point along its preferred easy axis direction $\hat{\mathbf{n}}$. Reducing the field past zero, the moments in the two side facets begin to rotate towards the field direction, as can be seen in the local distribution of $M_{n}$ in Fig. 7(A) $\left(M_{n} \approx\right.$ 0 in the side facets), where $M_{n}$ is the component of $\mathbf{M}$ pointing in the direction of the easy axis $\hat{\mathbf{n}}$. As with the other configurations, this configuration corresponds to the field value indicated by the corresponding letter in Fig. 6(c). Next, as indicated by two black arrows in Fig. 7(B), the magnetic moments in two of the side vertices rotate, leading to a strong negative spike in $\Delta f . \Delta f$ recovers for a small field range, shown as (C), until the moments in the other two side vertices rotate, leading to a somewhat smaller spike, (D). From here, $\Delta f$ further decreases, and the moments in the top and bottom facets rotate, as shown in (E). A last jump occurs as the moments in the top and bottom vertices reverse their $M_{n}$, depicted in (F). Finally, $(\mathrm{G})$ shows the distribution with which $M_{n}$ decreases as the magnetic moments complete their rotation towards $-\hat{\mathbf{z}}$. Although appearing in a slightly different field range and with smaller magnitude, the three spikes in the $\Delta f(H)$ curve simulated with $\mathrm{PBC}$ are also clearly observable in the experiment, cf., Figs. 6(a) and 6(c). This effect is likely due to the imperfect hexagonality of the measured nanotube. Note that for a perfect alignment $\left(\theta_{n}=90^{\circ}\right)$, one would expect the discontinuities shown at (B) and (D) to merge, since by symmetry the vertices shown in Figs. 7(B) and (D) must reverse at the same applied field.

In contrast to the the $\Delta f(H)$ curve simulated with PBCs, the curve simulated without PBCs has a single discontinuity, which occurs for a smaller applied field than the three spikes, as shown in Fig. 6(d). A second, even smaller discontinuity is seen just before crossing zero field. To shed light on the calculated progression of magnetic configurations, we show in Fig. 8 images of the magnetic configurations corresponding to the points indicated in Fig. 6(d).

Figure $8(\mathrm{~J})$ shows the tube magnetization saturated along $\hat{\mathbf{z}}$ at large positive field. As the field is reduced across (K) and then (L), we observe the staggered tilting of magnetic moments imposed by the differences in demagnetization field within the hexagonal cross section of the tube [Fig. 7(I)]. The behavior of the tube ends differs from that of the central part. Between $(\mathrm{L})$ and $(\mathrm{N})$, vortices nucleate at the ends, producing the corresponding discontinuity for small positive field in Fig. 6(d). At zero field $\mathrm{O}$, the vortices have maximally expanded along the tube, separated by a region of magnetic moments pointing along the preferred easy axis direction. This configuration is essentially the "twisted bamboo" state consisting of two opposing vortex configurations separated by a domain pointing along the tube axis. This configuration also

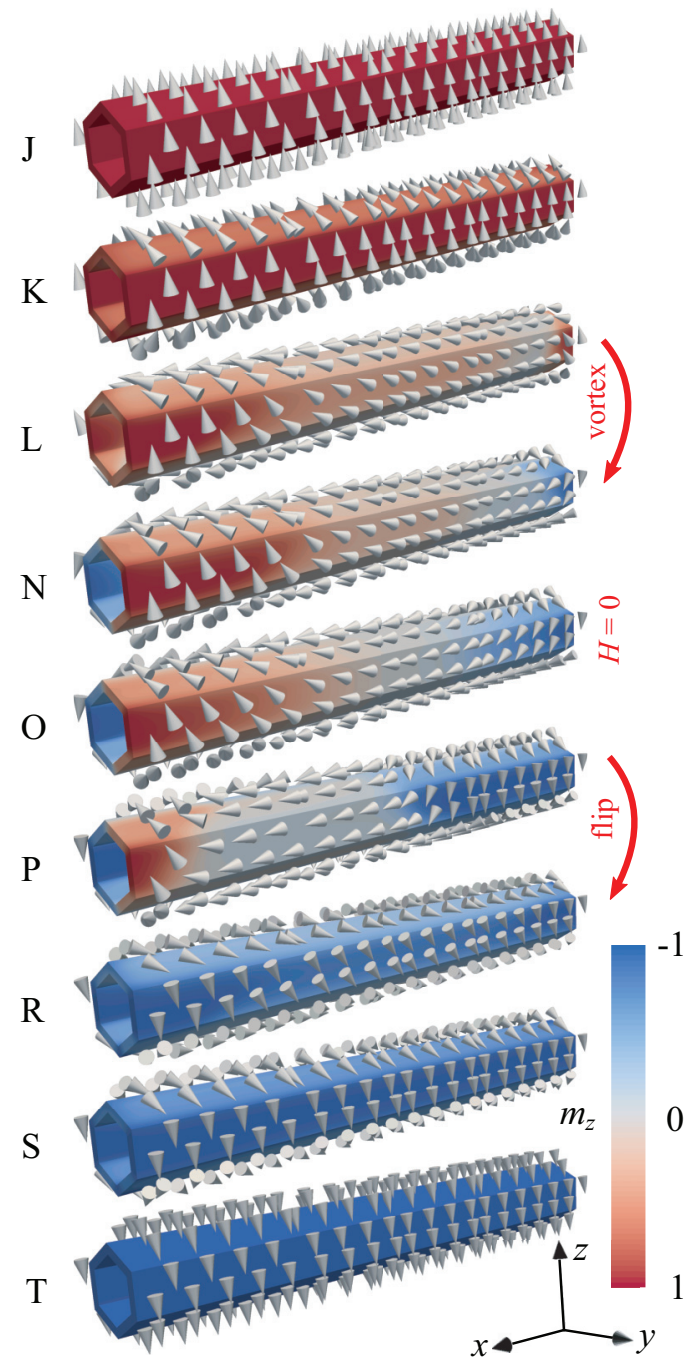

FIG. 8. Visualization of the magnetization configurations calculated without PBCs for nine different applied magnetic fields indicated in Fig. 6(d). The applied field is swept from pointing in $+\hat{\mathbf{z}}$ to $-\hat{\mathbf{z}}$ with $\hat{\mathbf{n}}$ pointing nearly along $\hat{\mathbf{x}}$ (configuration 2 or 3 ).

appeared near zero field in the simulations calculated without PBCs for configuration 1, i.e., Figs. 5(C) and 5(D). The only difference is that, in the present case, the domain parallel to the tube axis is more extended. In $\mathrm{P}$, the moments continue to rotate along the negative applied field. Now, however, since the preferred easy axis direction has reversed, the moments in the parallel domain find themselves pointing against the most energetically favorable direction. The vortices seem to stabilize the overall magnetic configuration, and, as a consequence, a single discontinuous change occurs between $\mathrm{P}$ and $\mathrm{R}$, resulting in a nearly uniform configuration without vortices, pointing mostly between the negative applied field and the preferred easy axis. $\mathrm{S}$ and $\mathrm{T}$ then show the final staggered rotation of the magnetic moments along the applied field. The simulated $\Delta f(H)$ curve corresponding to this progression strongly resembles the reversal measured by DCM shown in Fig. 6(b). The absence of the discontinuity due to the vortex nucleation in the measurement is likely due to the disproportionately 
large weight of the nanotube ends in the simulation, as already discussed in Sec. VI B 1.

c. Discussion. The two types of DCM reversals shown in Figs. 6(a) and 6(b) occur statistically and resemble simulations carried out with PBCs and without, respectively. Therefore, we hypothesize that the reversal in the perpendicular field geometry takes either the form of a staggered rotation without the formation of vortices [Fig. 6(a)] or a reversal in which vortices nucleate and a low-field "twisted bamboo" state is traversed [Fig. 6(b)] as depicted in Fig. 8.

\section{CONCLUSION}

To conclude, we investigate magnetic states of individual CoFeB nanotubes in an applied magnetic field at $4.2 \mathrm{~K}$ for different tube-to-field orientations using DCM. Single nanotubes are attached to the end of an ultrasoft cantilever and the shift of the cantilever's resonance frequency due to the dynamic magnetic torque is detected. We introduce an analytical model for DCM of an idealized SW magnet, in which all moments act in unison. Applying this model to the magnetometry data, we are able to describe behavior at high external fields and extract a saturation magnetization $\mu_{0} M_{s}=1.3 \pm 0.1 \mathrm{~T}$ for the CoFeB nanotubes.

In order to construct a more realistic model and to describe the behavior of the nanotubes at low field, we develop a numerical micromagnetic framework for calculating DCM frequency shifts and implement it using Nmag. These numerical simulations show that hysteresis loops measured by DCM in both parallel and-in some cases - in perpendicular applied field resemble what is expected for a reversal sequence nucleated by vortices at the tube ends. Such reversals include a "twisted bamboo" flux-closure configuration at low fields. Although the measurements in parallel field show the signature of a stable configuration near zero field, corresponding simulations do not confirm the behavior. This discrepancy may be due to the stabilizing effect of imperfections in real samples on magnetic configurations at low field.

Future work to confirm the presence and spatial character of these configurations should focus on noninvasive imaging of the nanotubes' magnetization. Potentially applicable techniques include scanning SQUID magnetometry, diamond NV center magnetometry [55], MFM [6,56], XMCDPEEM [11,57]. Efforts should also be made to produce more ideal ferromagnetic nanotubes, including cutting flat ends using focused ion beam milling or removing antiferromagnetic surface oxides. Finally, we note that our numerical method for calculating DCM response is not specific to ferromagnetic nanotubes and is therefore applicable to a broad range of nanomagnetic samples. The framework may allow us to accurately interpret the DCM response of other nanometer-scale ferromagnets or even of antiferromagnets and helimagnets.

\section{ACKNOWLEDGMENTS}

We thank Gözde Tütüncüoglu and Federico Matteini for providing the GaAs NW used as templates for the $\mathrm{CoFeB}$ nanotubes. We gratefully acknowledge financial support from the Canton Aargau, the Swiss Nanoscience Institute (SNI), the Swiss National Science Foundation (SNSF) under Grant No.
200020-159893, the NCCR Quantum Science and Technology (QSIT), and the Deutsche Forschungsgemeinschaft via project GR1640/5-2 (Priority Program SPP 1538).

\section{APPENDIX A: ANALYTICAL APPROXIMATION FOR DCM FREQUENCY SHIFT}

In the SW model, the magnetic energy $E_{m}$ and the magnetic history of the magnet determine the equilibrium magnetization M. $E_{m}$ in turn depends on the external field $\mathbf{H}$ and the magnet's properties. In order to calculate $E_{m}$, we define the different orientations of the nanotube in our coordinate system, as depicted in Fig. 1, with the spherical coordinates $\theta_{n}$ and $\phi_{n}$. Likewise, we describe the net magnetization $\mathbf{M}$ with the angles $\phi_{m}$ and $\theta_{m}$, so that

$$
\mathbf{M}=M_{s}\left(\begin{array}{c}
\sin \theta_{m} \cos \phi_{m} \\
\sin \theta_{m} \sin \phi_{m} \\
\cos \theta_{m}
\end{array}\right),
$$

where $M_{s}$ is a constant. In this approximation, the magnetic energy consists of a Zeeman term and an anisotropy term:

$$
E_{m}=-\mu_{0} V \mathbf{M} \cdot \mathbf{H}+\frac{1}{2} \mu_{0} V D_{u}(\mathbf{M} \cdot \hat{\mathbf{n}})^{2},
$$

where $\mu_{0}$ is the vacuum permeability and $V$ is the volume of the nanotube. Therefore, $E_{m}$ depends on $\mathbf{H}, \phi_{m}, \theta_{m}, \phi_{n}$, and $\theta_{n}$. In order to determine the behavior of $\mathbf{M}$ in our experiment, we introduce the oscillating cantilever. Oscillation of the cantilever amounts to a rotation of the nanotube orientation $\hat{\mathbf{n}}$ about $\hat{\mathbf{y}}$. This process introduces an additional $\theta_{c}$ dependence to the nanotube orientation and thus also to $E_{m}$.

Since the microscopic processes in ferromagnetic nanotubes are expected to be much faster than the cantilever resonance frequency $[10,38,40]$, the magnetization of the nanotube can always be assumed to be in its equilibrium orientation. We can therefore solve for $\phi_{m}$ and $\theta_{m}$, by fulfilling the following minimization conditions for $E_{m}$ :

$$
\begin{gathered}
\frac{\partial E_{m}}{\partial \phi_{m}}=\frac{\partial E_{m}}{\partial \theta_{m}}=0 ; \\
\frac{\partial^{2} E_{m}}{\partial \phi_{m}^{2}}, \quad \frac{\partial^{2} E_{m}}{\partial \theta_{m}^{2}}>0 .
\end{gathered}
$$

Since $\theta_{c} \ll 1$, we approximate the solutions for $\phi_{m}$ and $\theta_{m}$ by considering only terms up to first order in $\theta_{c}$ :

$$
\begin{gathered}
\phi_{m}\left(\theta_{c}\right) \approx \phi_{m}(0)+\left.\frac{\partial \phi_{m}}{\partial \theta_{c}}\right|_{\theta_{c}=0} \cdot \theta_{c} \\
\theta_{m}\left(\theta_{c}\right) \approx \theta_{m}(0)+\left.\frac{\partial \theta_{m}}{\partial \theta_{c}}\right|_{\theta_{c}=0} \cdot \theta_{c} .
\end{gathered}
$$

As expected, once solutions of this form are found, we see that the equilibrium angles of the magnetization for $\theta_{c}=0$ give the solutions already known from the SW model [61]. $\phi_{m}(0)=\phi_{n}$, such that the azimuthal orientation of the magnetization always follows the azimuthal orientation of the magnet. In other words, the magnetization is constrained to the plane defined by the magnet's uniaxial anisotropy axis and the direction of the magnetic field. $\theta_{m}(0)$ is given by the arctangent of a solution to a quartic equation. Either one or two of the 

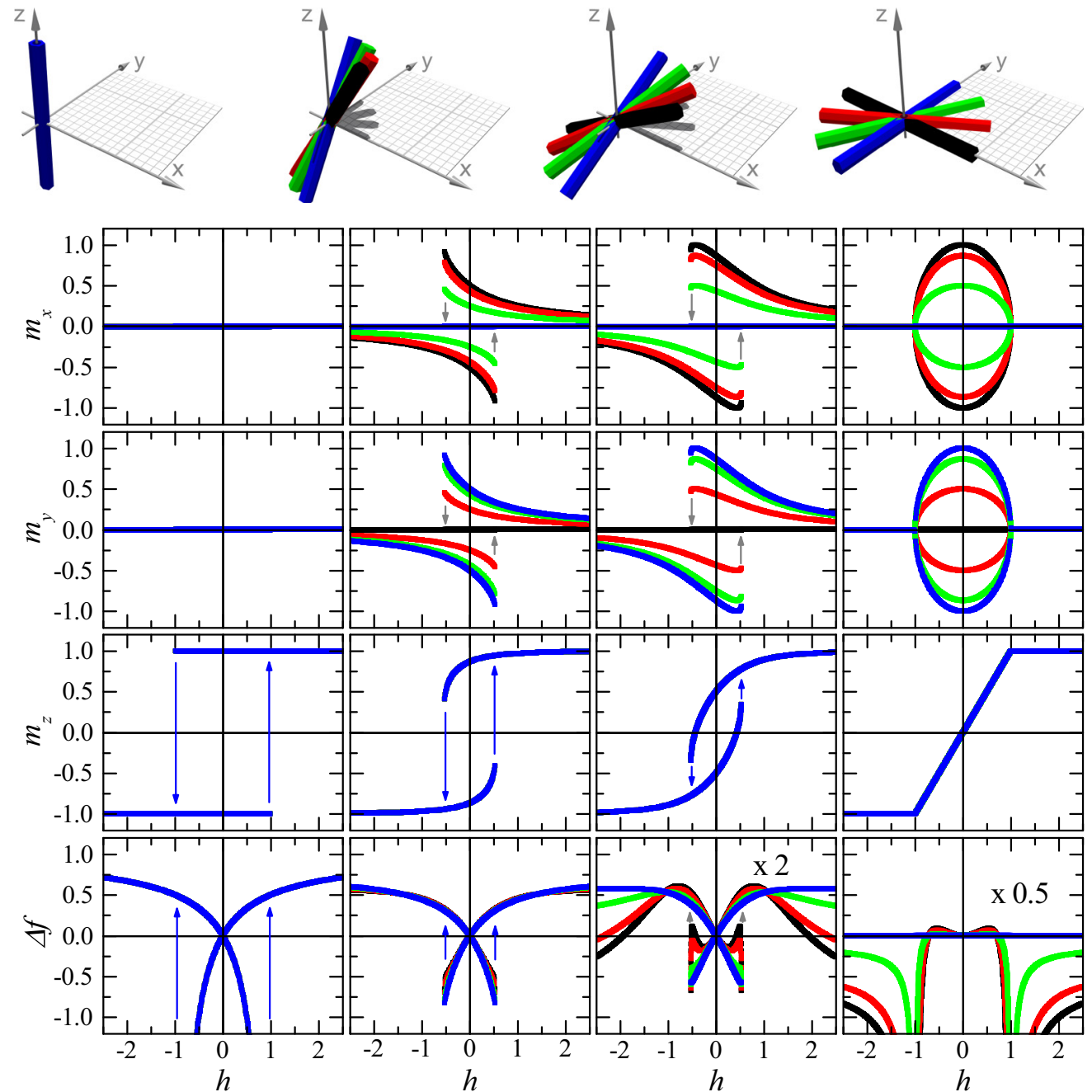

FIG. 9. Components of $\mathbf{M}$ normalized by $M_{s}$ (first three rows) and $\Delta f$ normalized by $\frac{f_{0} \mu_{0} V M_{s}^{2}}{2 k_{0} l_{e}^{2}}$ (last row) vs normalized magnetic field $h=-\frac{H}{M_{s} D_{u}}$ for different orientations of the nanotubes. $\theta_{n}$ is increased from $0^{\circ}$ in the first column by $30^{\circ}$ per column up to $90^{\circ} . \phi_{m}$ is changed in the same steps, given by the different, color-coded graphs within each column. Arrows indicate switching of the magnetization.

four possible solutions for $\theta_{m}(0)$ are real and minimize $E_{m}$. When there are two physical solutions, the system allows for magnetic hysteresis. In the first three rows of Fig. 9, we plot the components of the resulting equilibrium magnetization $\mathbf{M}$ normalized to $M_{s}$ as a function of the reduced magnetic field $h=-\frac{H}{M_{s} D_{u}}$ for $\theta_{c}=0$ and several orientations $\phi_{n}$ and $\theta_{n}$.

Solutions for $\phi_{m}\left(\theta_{c}\right)$ and $\theta_{m}\left(\theta_{c}\right)$ in the form of (A5) and (A6) are then replaced into the expression for $E_{m}$. By taking the second derivative of $E_{m}$ with respect to $\theta_{c}$ and applying (5), we find the corresponding frequency shift measured by DCM. We implement the above procedure in a Mathematica script, which allows us to determine the magnetization and corresponding frequency shift for any orientation of the nanotube $\left(\phi_{n}, \theta_{n}\right)$ in any applied magnetic field. The last row of Fig. 9 shows the corresponding frequency shift measured by DCM, $\Delta f$, as a function of $h$ and normalized to $\frac{f_{0} \mu_{0} V M_{s}^{2}}{2 k_{0} l_{e}^{2}}$.

\section{The high-field limit}

Regardless of the nanotube's orientation, for large fields, i.e., $H \gg\left|D_{u} M_{s}\right|, \mathbf{M}$ is forced to be parallel to $\mathbf{H}$. By applying this limit to (A2) and solving (5), we find that $\Delta f$ approaches a horizontal asymptote given by:

$$
\Delta f=\frac{f_{0} \mu_{0} V}{2 k_{0} l_{e}^{2}} M_{s}^{2} D_{u}\left(\sin ^{2} \theta_{n} \cos ^{2} \phi_{n}-\cos ^{2} \theta_{n}\right) .
$$

In this limit, $\Delta f$ is a measure of the anisotropy energy of the nanomagnetic particle, multiplied by a factor depending on its orientation relative to $\mathbf{H}$. In short, by forcing $\mathbf{M}$ and $\mathbf{H}$ to be parallel, the cantilever oscillation only probes the curvature of the second term in the magnetic energy shown in (A2). For example, as can be seen from (A7) and the last row of Fig. 9, at high field, a nanotube oriented along $\theta_{n}=0$ will approach an equal and opposite $\Delta f$ as when it is oriented along $\phi_{n}=0$ and $\theta_{n}=90^{\circ}$. When the anisotropy axis $\hat{\mathbf{n}}$ of an idealized nanotube is coincident with the axis of cantilever oscillation $\hat{\mathbf{y}}\left(\phi_{n}=\right.$ $\left.\theta_{n}=90^{\circ}\right), \Delta f=0$. The cantilever frequency is unaffected in this geometry, because the idealized cylindrical nanotube is symmetric about $\hat{\mathbf{n}}$. Since $\hat{\mathbf{n}} \| \hat{\mathbf{y}}$, the magnetic energy has no curvature along $\theta_{c}$. 


\section{Intermediate fields}

At intermediate fields, we can understand the relationship between $\Delta f(H)$ curves and the $\mathrm{SW}$ magnetization curves by considering the limiting orientations. The first column of Fig. 9 shows the case of a nanotube with its easy axis aligned along $\mathbf{H}\left(\theta_{n}=0\right)$. The magnetization $M_{z}$ executes the expected square hysteresis loop as a function of $H$, while $M_{x}=M_{y}=0$. As $H$ is swept down from high fields, $\mathbf{M}$ is parallel to $\mathbf{H}$, making the physical orientation of the nanotube in the $x z$ plane energetically optimal. As a result, $\Delta f>0$. Upon crossing $H=0$, however, the direction of $\mathbf{H}$ inverts and $\mathbf{M}$ becomes antiparallel to $\mathbf{H}$, making the nanotube's orientation energetically unfavorable. As a result, $\Delta f<0$. Once the coercive field is reached and $\mathbf{M}$ again switches into a parallel orientation with respect to $\mathbf{H}$, the frequency shift also switches sign giving $\Delta f>0$.

The last column of Fig. 9 shows the case of a nanotube with its easy axis aligned perpendicular to $\mathbf{H}\left(\theta_{n}=90^{\circ}\right)$. As $H$ is increased across zero, the magnetization $\mathbf{M}$ rotates coherently from alignment with $-\hat{\mathbf{z}}$ through the $x y$ plane to $+\hat{\mathbf{z}}$ without hysteresis. As $H$ is swept down from high fields, $\mathbf{M}$ is parallel to $\mathbf{H}$ and perpendicular to the anisotropy axis, making the physical orientation of the nanotube energetically unfavorable and giving $\Delta f<0$. Once $H$ is reduced enough that $\mathbf{M}$ has tilted closer to the $x y$ plane than to $\hat{\mathbf{z}}$ (i.e., $\theta_{m}>45^{\circ}$ ), the orientation of the nanotube becomes energetically favorable and $\Delta f>0$. As $H$ decreases across zero, the behavior is symmetric.

Intermediate orientations depicted in the middle columns of Fig. 9 show the effects of arbitrary alignments of the nanotube with respect to $\mathbf{H}$. In general, alignments between $\hat{\mathbf{n}} \| \mathbf{H}$ and $\hat{\mathbf{n}} \perp \mathbf{H}$ reduce the expected $|\Delta f|$. Even a slight misalignment from the $\hat{\mathbf{n}} \perp \mathbf{H}$ case, introduces hysteresis to the magnetization loop, since the energetic symmetry of $\pm \hat{\mathbf{n}}$ is broken by $\mathbf{H}$.

Note that for all orientations at $H=0, \Delta f=0$. Indeed, the effects that we observe all arise due to the interaction between the magnetization of the nanotube and the externally applied field $\mathbf{H}$. A curvature in $E_{m}$ exists with respect to $\theta_{c}$ only because $\theta_{c}$ changes the nanotube's orientation relative to $\mathbf{H}$; when $H=0$, these interactions vanish. As a result, the sensitivity of DCM becomes progressively worse as $\mathbf{H}$ approaches zero, at which point the technique is completely insensitive. Jang et al. describe a variation on DCM, known as phase-locked cantilever magnetometry (PLCM), with the additional application of an AC magnetic field in order to overcome this limitation [62].

\section{The low-field limit}

For low applied magnetic fields, i.e., $H \ll\left|D_{u} M_{s}\right|$, shape anisotropy dominates the magnetic energy in the SW model and ensures that the net magnetization $\mathbf{M}$ remains either parallel or antiparallel to the nanotube axis $\hat{\mathbf{n}}$. In this case, the cantilever oscillation only probes the curvature of the first term in the magnetic energy shown in (A2). Applying this limit to (A2) and solving (5), we find:

$$
\Delta f=\frac{f_{0} \mu_{0} V}{2 k_{0} l_{e}^{2}} H M_{s} \cos \theta_{n}=\frac{f_{0} \mu_{0} V}{2 k_{0} l_{e}^{2}} H M_{z} .
$$

This low-field regime constitutes a special case, since this expression allows the direct determination of $M_{z}$ from measurements of $\Delta f$. By solving (A8) for $M_{z}$, at low field we have:

$$
M_{z}=\frac{2 k_{0} l_{e}^{2}}{f_{0} \mu_{0} V H} \Delta f
$$

Despite the fact that nonuniform magnetization configurations are likely present in the nanotubes at low field, these equations allow us to analyze DCM data and extract an effective magnetization that describes the behavior of an equivalent SW magnet. Such analysis allows us to see the extent to which the magnetic configuration within the nanotube is uniform and rotates coherently.

\section{APPENDIX B: NUMERICAL CALCULATION OF DCM FREQUENCY SHIFT}

We employ the following procedure in order to calculate the DCM frequency shift from the Nmag simulations:

(1) For each value of the external magnetic field $H$, we calculate the magnetization configuration with Nmag for the cantilever in its equilibrium orientation $\theta_{c}=0$.

(2) We then calculate the magnetization $\mathbf{m}_{i}$ at the centroid $\mathbf{r}_{i}$ of each tetrahedral mesh element $i$. We use a Shepard weighting $w_{j}\left(\mathbf{r}_{j}\right)=\left|\mathbf{r}_{i}-\mathbf{r}_{j}\right|^{-2}$, where the indices refer to the $j$ th vertex of the $i$ th tetrahedron, to obtain

$$
\mathbf{m}_{i}=\frac{\sum_{j} w_{j} \mathbf{m}_{j}}{\sum_{j} w_{j}}
$$

This calculation is necessary, since Nmag determines the magnetization at the vertices of the tetrahedra only.

(3) We calculate the magnetic moment $\mu_{i}$ of each tetrahedron via $\boldsymbol{\mu}_{i}=\mathbf{m}_{i} V_{i}$, with the volume $V_{i}$ of the corresponding tetrahedron. The total magnetic moment of the tube is then $\boldsymbol{\mu}_{\text {tube }}=\sum_{i} \boldsymbol{\mu}_{i}$. The resultant magnetic torque on the tube along the cantilever's axis of rotation is given by

$$
\tau_{m, y}=\left(\boldsymbol{\mu}_{\text {tube }} \times \mu_{0} \mathbf{H}\right) \cdot \hat{\mathbf{y}} .
$$

(4) Having calculated the magnetic torque on the tube for each value of $H$ in the field sweep at $\theta_{c}=0$, we now tilt the tube by a small but finite angle $\delta \theta_{c} \leqslant 0.3^{\circ}$ and repeat the three preceding steps. Now in addition to $\tau_{m, y}(0)$, we obtain $\tau_{m, y}\left(\delta \theta_{c}\right)$.

(5) We then find the DCM frequency shift using $\tau_{m, y}=$ $-\partial E_{m} / \partial \theta_{c}$ and (5):

$$
\begin{aligned}
\Delta f & =\frac{f_{0}}{2 k_{0} l_{e}^{2}}\left(-\left.\frac{\partial \tau_{m, y}}{\partial \theta_{c}}\right|_{\theta_{c}=0}\right) \\
& \approx-\frac{f_{0}}{2 k_{0} l_{e}^{2}} \frac{\tau_{m, y}\left(\delta \theta_{c}\right)-\tau_{m, y}(0)}{\delta \theta_{c}} .
\end{aligned}
$$


[1] S. S. P. Parkin, M. Hayashi, and L. Thomas, Science 320, 190 (2008).

[2] M. M. Maqableh, X. Huang, S.-Y. Sung, K. S. M. Reddy, G. Norby, R. H. Victora, and B. J. H. Stadler, Nano Lett. 12, 4102 (2012).

[3] S. Khizroev, M. H. Kryder, D. Litvinov, and D. A. Thompson, Appl. Phys. Lett. 81, 2256 (2002).

[4] M. Poggio and C. L. Degen, Nanotechnology 21, 342001 (2010).

[5] H. Campanella, M. Jaafar, J. Llobet, J. Esteve, M. Vázquez, A. Asenjo, R. P. d. Real, and J. A. Plaza, Nanotechnology 22, 505301 (2011).

[6] F. J. Castaño, C. A. Ross, C. Frandsen, A. Eilez, D. Gil, H. I. Smith, M. Redjdal, and F. B. Humphrey, Phys. Rev. B 67, 184425 (2003).

[7] Z. K. Wang, H. S. Lim, H. Y. Liu, S. C. Ng, M. H. Kuok, L. L. Tay, D. J. Lockwood, M. G. Cottam, K. L. Hobbs, P. R. Larson, J. C. Keay, G. D. Lian, and M. B. Johnson, Phys. Rev. Lett. 94, 137208 (2005).

[8] J. Topp, J. Podbielski, D. Heitmann, and D. Grundler, Phys. Rev. B 78, 024431 (2008).

[9] P. Landeros, O. J. Suarez, A. Cuchillo, and P. Vargas, Phys. Rev. B 79, 024404 (2009).

[10] P. Landeros and 1. S. Núñez, J. Appl. Phys. 108, 033917 (2010).

[11] R. Streubel, V. P. Kravchuk, D. D. Sheka, D. Makarov, F. Kronast, O. G. Schmidt, and Y. Gaididei, Appl. Phys. Lett. 101, 132419 (2012).

[12] D. Rüffer, R. Huber, P. Berberich, S. Albert, E. Russo-Averchi, M. Heiss, J. Arbiol, A. F. i. Morral, and D. Grundler, Nanoscale 4, 4989 (2012).

[13] A. Buchter, J. Nagel, D. Rüffer, F. Xue, D. P. Weber, O. F. Kieler, T. Weimann, J. Kohlmann, A. B. Zorin, E. Russo-Averchi, R. Huber, P. Berberich, A. Fontcuberta i Morral, M. Kemmler, R. Kleiner, D. Koelle, D. Grundler, and M. Poggio, Phys. Rev. Lett. 111, 067202 (2013).

[14] R. Streubel, J. Lee, D. Makarov, M.-Y. Im, D. Karnaushenko, L. Han, R. Schäfer, P. Fischer, S.-K. Kim, and O. G. Schmidt, Adv. Mater. 26, 316 (2014).

[15] P. Landeros, S. Allende, J. Escrig, E. Salcedo, D. Altbir, and E. E. Vogel, Appl. Phys. Lett. 90, 102501 (2007).

[16] J. Escrig, J. Bachmann, J. Jing, M. Daub, D. Altbir, and K. Nielsch, Phys. Rev. B 77, 214421 (2008).

[17] J. Bachmann, J. Escrig, K. Pitzschel, J. M. M. Moreno, J. Jing, D. Görlitz, D. Altbir, and K. Nielsch, J. Appl. Phys. 105, 07B521 (2009).

[18] O. Albrecht, R. Zierold, S. Allende, J. Escrig, C. Patzig, B. Rauschenbach, K. Nielsch, and D. Görlitz, J. Appl. Phys. 109, 093910 (2011).

[19] R. Streubel, D. Makarov, J. Lee, C. MüLler, M. Melzer, R. SchäFer, C. C. B. Bufon, S.-K. Kim, and O. G. Schmidt, SPIN 3, 1340001 (2013).

[20] J. Bachmann, Jing, M. Knez, S. Barth, H. Shen, S. Mathur, U. Gösele, and K. Nielsch, J. Am. Chem. Soc. 129, 9554 (2007).

[21] M. Daub, M. Knez, U. Goesele, and K. Nielsch, J. Appl. Phys. 101, 09J111 (2007).

[22] A. Rudolph, M. Soda, M. Kiessling, T. Wojtowicz, D. Schuh, W. Wegscheider, J. Zweck, C. Back, and E. Reiger, Nano Lett. 9, 3860 (2009).
[23] Y. T. Chong, D. Görlitz, S. Martens, M. Y. E. Yau, S. Allende, J. Bachmann, and K. Nielsch, Adv. Mater. 22, 2435 (2010).

[24] C. Rossel, P. Bauer, D. Zech, J. Hofer, M. Willemin, and H. Keller, J. Appl. Phys. 79, 8166 (1996).

[25] J. G. E. Harris, D. D. Awschalom, F. Matsukura, H. Ohno, K. D. Maranowski, and A. C. Gossard, Appl. Phys. Lett. 75, 1140 (1999).

[26] B. C. Stipe, H. J. Mamin, T. D. Stowe, T. W. Kenny, and D. Rugar, Phys. Rev. Lett. 86, 2874 (2001).

[27] A. C. Bleszynski-Jayich, W. E. Shanks, B. Peaudecerf, E. Ginossar, F. v. Oppen, L. Glazman, and J. G. E. Harris, Science 326, 272 (2009).

[28] J. Jang, D. G. Ferguson, V. Vakaryuk, R. Budakian, S. B. Chung, P. M. Goldbart, and Y. Maeno, Science 331, 186 (2011).

[29] L. Bossoni, P. Carretta, and M. Poggio, Appl. Phys. Lett. 104, 182601 (2014).

[30] P. Banerjee, F. Wolny, D. V. Pelekhov, M. R. Herman, K. C. Fong, U. Weissker, T. Mühl, Y. Obukhov, A. Leonhardt, B. Büchner, and P. C. Hammel, Appl. Phys. Lett. 96, 252505 (2010).

[31] D. P. Weber, D. Rüffer, A. Buchter, F. Xue, E. Russo-Averchi, R. Huber, P. Berberich, J. Arbiol, A. Fontcuberta i Morral, D. Grundler, and M. Poggio, Nano Lett. 12, 6139 (2012).

[32] A. Mehlin, F. Xue, D. Liang, H. F. Du, M. J. Stolt, S. Jin, M. L. Tian, and M. Poggio, Nano Lett. 15, 4839 (2015).

[33] R. Hertel and J. Kirschner, J. Magn. Magn. Mater. 278, L291 (2004).

[34] J. Escrig, P. Landeros, D. Altbir, E. E. Vogel, and P. Vargas, J. Magn. Magn. Mater. 308, 233 (2007).

[35] J. Escrig, P. Landeros, D. Altbir, and E. E. Vogel, J. Magn. Magn. Mater. 310, 2448 (2007).

[36] A. P. Chen, K. Y. Guslienko, and J. Gonzalez, J. Appl. Phys. 108, 083920 (2010).

[37] A.-P. Chen, J. M. Gonzalez, and K. Y. Guslienko, J. Appl. Phys. 109, 073923 (2011).

[38] M. Yan, C. Andreas, A. Kákay, F. García-Sánchez, and R. Hertel, Appl. Phys. Lett. 100, 252401 (2012).

[39] D. Rüffer, M. Slot, R. Huber, T. Schwarze, F. Heimbach, G. Tütüncüoglu, F. Matteini, E. Russo-Averchi, A. Kovács, R. Dunin-Borkowski, R. R. Zamani, J. R. Morante, J. Arbiol, A. F. i. Morral, and D. Grundler, APL Materials 2, 076112 (2014).

[40] T. Schwarze and D. Grundler, Appl. Phys. Lett. 102, 222412 (2013).

[41] A. T. Hindmarch, C. J. Kinane, M. MacKenzie, J. N. Chapman, M. Henini, D. Taylor, D. A. Arena, J. Dvorak, B. J. Hickey, and C. H. Marrows, Phys. Rev. Lett. 100, 117201 (2008).

[42] H. Ulrichs, B. Lenk, and M. Münzenberg, Appl. Phys. Lett. 97, 092506 (2010).

[43] D. Rugar, H. J. Mamin, and P. Guethner, Appl. Phys. Lett. 55, 2588 (1989).

[44] A. Aharoni, Introduction to the Theory of Ferromagnetism (Oxford University Press, Oxford, 2000).

[45] R. Skomski and J. M. D. Coey, Permanent Magnetism (Institute of Physics Publishing, Bristol, 1999).

[46] E. C. Stoner, Philosophical Magazine Series 7 36, 803 (1945).

[47] W. F. Brown and A. H. Morrish, Phys. Rev. 105, 1198 (1957).

[48] M. Beleggia, D. Vokoun, and M. De Graef, J. Magn. Magn. Mater. 321, 1306 (2009). 
[49] J. G. E. Harris, Ph.D. thesis, University of California, Santa Barbara, California, 2000.

[50] E. Straver, Ph.D. thesis, Stanford University, Stanford, California, 2004.

[51] A. Kamra, M. Schreier, H. Huebl, and S. T. B. Goennenwein, Phys. Rev. B 89, 184406 (2014).

[52] T. Fischbacher, M. Franchin, G. Bordignon, and H. Fangohr, IEEE Transactions on Magnetics 43, 2896 (2007).

[53] C. Bilzer, T. Devolder, J.-V. Kim, G. Counil, C. Chappert, S. Cardoso, and P. P. Freitas, J. Appl. Phys. 100, 053903 (2006).

[54] M. Montinaro, Ph.D. thesis, Universität Basel, Basel, Switzerland, 2014.

[55] L. Rondin, J.-P. Tetienne, T. Hingant, J.-F. Roch, P. Maletinsky, and V. Jacques, Rep. Prog. Phys. 77, 056503 (2014).

[56] S. P. Li, D. Peyrade, M. Natali, A. Lebib, Y. Chen, U. Ebels, L. D. Buda, and K. Ounadjela, Phys. Rev. Lett. 86, 1102 (2001).
[57] R. Streubel, D. J. Thurmer, D. Makarov, F. Kronast, T. Kosub, V. Kravchuk, D. D. Sheka, Y. Gaididei, R. Schäfer, and O. G. Schmidt, Nano Lett. 12, 3961 (2012).

[58] A. Buchter, R. Wölbing, M. Wyss, O. F. Kieler, T. Weimann, J. Kohlmann, A. B. Zorin, D. Rüffer, F. Matteini, G. Tütüncüoglu, F. Heimbach, A. Kleibert, A. Fontcuberta i Morral, D. Grundler, R. Kleiner, D. Koelle, and M. Poggio, Phys. Rev. B 92, 214432 (2015).

[59] F. J. Castaño, C. A. Ross, A. Eilez, W. Jung, and C. Frandsen, Phys. Rev. B 69, 144421 (2004).

[60] T. J. Klemmer, K. A. Ellis, L. H. Chen, B. van Dover, and S. Jin, J. Appl. Phys. 87, 830 (2000).

[61] A. H. Morrish, The Physical Principles of Magnetism (IEEE Press, New York, 2001).

[62] J. Jang, R. Budakian, and Y. Maeno, Appl. Phys. Lett. 98, 132510 (2011). 